\title{
Evaluating a Staff-Child Interaction Therapy Workshop for Home- Based Mental Health Providers: Effects on Therapist Skill Change
}

Cree Robinson

Follow this and additional works at: https://researchrepository.wvu.edu/etd

\section{Recommended Citation}

Robinson, Cree, "Evaluating a Staff-Child Interaction Therapy Workshop for Home-Based Mental Health Providers: Effects on Therapist Skill Change" (2016). Graduate Theses, Dissertations, and Problem Reports. 6517.

https://researchrepository.wvu.edu/etd/6517

This Thesis is protected by copyright and/or related rights. It has been brought to you by the The Research Repository @ WVU with permission from the rights-holder(s). You are free to use this Thesis in any way that is permitted by the copyright and related rights legislation that applies to your use. For other uses you must obtain permission from the rights-holder(s) directly, unless additional rights are indicated by a Creative Commons license in the record and/ or on the work itself. This Thesis has been accepted for inclusion in WVU Graduate Theses, Dissertations, and Problem Reports collection by an authorized administrator of The Research Repository @ WVU. For more information, please contact researchrepository@mail.wvu.edu. 
Evaluating a Staff-Child Interaction Therapy Workshop for Home-Based Mental Health Providers: Effects on Therapist Skill Change

Cree Robinson, B.A.

\author{
Thesis submitted to \\ the Eberly College of Arts and Sciences \\ at West Virginia University \\ in partial fulfillment of the requirements \\ for the degree of \\ Master of Science \\ in \\ Psychology
}

Cheryl B. McNeil, Ph.D., Chair

Melissa Blank, Ph.D.

Christina Duncan, Ph.D.

Department of Psychology

Morgantown, West Virginia

2016

Keywords: Staff-Child Interaction Therapy, Wraparound, child behavior problems, staff training

Copyright 2016 Cree Robinson 


\begin{abstract}
Evaluating a Staff-Child Interaction Therapy Workshop for Home-Based Mental Health Providers: Effects on Therapist Skill Change
\end{abstract}

Cree Robinson, B.A.

The current study evaluated the effectiveness of a one-day training workshop used to train bachelor's level mental health staff in Staff-Child Interaction Therapy (SCIT), a newly developed intervention designed to reduce problem behaviors in children 2- to -9 years of age through the use of behavioral management techniques by home-based staff. A total of 39 therapists, known as Therapeutic Support Staff (TSS), were involved in this study, 20 who received training in SCIT (i.e., experimental group) and 19 who received training in Compassion Fatigue (i.e., attention control group). This study was a part of a larger outcome study that included additional training workshops and an evaluation of the SCIT protocol for changing child behavior problems in the home. Therapists in both groups completed a baseline assessment at the beginning of the workshop day. To measure the skills, therapists interacted with confederate children (adult research assistants who were trained to act like children) during two 5-minute situations (ChildLed Play and Clean-Up). These interactions were live coded by a trained research assistant using the Dyadic Parent-Child Interaction Coding System (DPICS; Eyberg, Nelson, Duke, \& Boggs, 2005) to measure the staff skill change. Therapists also completed the TSS Demographic Form at baseline. Approximately 6-to-8 weeks following the baseline assessment, therapists completed a post-training assessment, which involved the same procedures as the baseline assessment. Analyses indicated that therapists who were trained in SCIT provided significantly more labeled praises, reflections, and behavior descriptions from pre-to post-assessment and significantly fewer questions, criticisms, and commands than therapists who received a Compassion Fatigue training workshop during the Child-Led Play DPICS situation. Regarding the Clean-Up DPICS situation, analyses indicated that therapists who were trained in SCIT provided significantly fewer questions and criticisms than therapists who received a Compassion Fatigue training workshop. There were no significant differences between the SCIT and Compassion Fatigue groups on any other dependent variables (e.g., contingent praise, effective commands). TSS from the study had difficulty learning two distinct sets of skills simultaneously. Specifically, it was challenging for TSS to master both ChildDirected Interaction skills (designed to improve TSS-child relationships) and AdultDirected Interaction skills (designed to help therapists implement effective discipline strategies) during a one-day workshop where they received only 4.5 actual hours of instruction. In future research, it may be more effective to teach positive relationship skills first and allow TSS to practice those skills before teaching discipline skills. This would allow therapists to have additional time to practice both skill sets, thus also improving their chances of meeting mastery criteria for two sets of disparate techniques. Overall, however, findings supported the use of this experiential, mastery-based workshop to teach bachelor's-level staff to manage problem behaviors in their child clients. The workshop resulted in many therapists mastering their skills at the end of the day of training and demonstrating sustained skill improvements 6-to- 8 weeks after the initial workshop. 
Table of Contents

Introduction $\quad 1$

$\begin{array}{lr}\text { Purpose of the Present Study } & 9\end{array}$

$\begin{array}{ll}\text { Study Hypotheses } & 10\end{array}$

$\begin{array}{ll}\text { Methodology } & 11\end{array}$

$\begin{array}{ll}\text { Participants } & 11\end{array}$

$\begin{array}{ll}\text { Baseline Assessment } & 13\end{array}$

$\begin{array}{ll}\text { Post-Training Assessment } & 15\end{array}$

$\begin{array}{ll}\text { Training and Coding of Observational } & 15\end{array}$

$\begin{array}{ll}\text { Control Workshop } & 17\end{array}$

$\begin{array}{ll}\text { SCIT Workshop } & 18\end{array}$

$\begin{array}{ll}\text { Mastery Criteria } & 20\end{array}$

$\begin{array}{ll}\text { Measures } & 21\end{array}$

$\begin{array}{ll}\text { TSS Demographic Form } & 21\end{array}$

Dyadic Parent-Child Interaction Coding System 21

$\begin{array}{ll}\text { Power Analysis } & 22\end{array}$

Interrater Reliability 22

$\begin{array}{ll}\text { Results } & 23\end{array}$

$\begin{array}{ll}\text { Preliminary Analyses } & 23\end{array}$

Results for Therapist Positive Composite 24

Results for Therapist Negative Composite 25

Results for Effective Commands 25 
Discussion

$\begin{array}{ll}\text { References } & 36\end{array}$

$\begin{array}{ll}\text { Table } 1 & 42\end{array}$

$\begin{array}{ll}\text { Table } 2 & 44\end{array}$

$\begin{array}{ll}\text { Table } 3 & 46\end{array}$

$\begin{array}{ll}\text { Table } 4 & 47\end{array}$

$\begin{array}{ll}\text { Table } 5 & 48\end{array}$

$\begin{array}{ll}\text { Table } 6 & 49\end{array}$

$\begin{array}{lr}\text { Table } 7 & 50\end{array}$

$\begin{array}{ll}\text { Figure } 1 & 52\end{array}$

$\begin{array}{ll}\text { Figure } 2 & 53\end{array}$

$\begin{array}{lr}\text { Figure } 3 & 54\end{array}$

Appendix A: SCIT vs. PCIT

Appendix B: ADI Diagram with ADI Script 56

Appendix C: SCIT Workshop Target Skills 57

Appendix D: DPICS Coding Sheet 58

Appendix E: Child Behavior Checklist 59

Appendix F: Treatment as Usual Discussion Questions 60

Appendix G: Control Workshop Protocol 61

Appendix H: SCIT Workshop Protocol 62

$\begin{array}{ll}\text { Appendix I: Mastery Criteria } & 63\end{array}$

Appendix J: TSS Demographic Form $\quad 64$

Appendix K: Variables Coded During Standard DPICS Situations 67 
Appendix L: Instructions for the Two Standard DPICS Observations 
Evaluating a Staff-Child Interaction Therapy Workshop for Home-Based Mental Health Providers: Effects on Therapist Skill Change

It is estimated that nearly 4.5 million children in the United States experience severe emotional and behavioral problems. However, most of them receive either inadequate mental health services or no treatment at all (National Institute for Health Care Management Research and Educational Foundation, 2005). Furthermore, when services are delivered to these children, they are typically limited in variety and have unknown effects. For example, in Pennsylvania, the site of the current study, individualized community-based mental health services, or "Wraparound programs," are offered to children and adolescents with severe emotional and behavioral disorders (Pennsylvania Department of Public Welfare, 2001). While these services are widespread throughout the state, research has shown the evidence base for Wraparound to be less than promising (Farmer, Dorsey \& Mustillo, 2004). Furthermore, the few studies that have investigated the outcomes of Wraparound possess major methodological concerns resulting in mixed and inconsistent findings (Bickman, Smith, Lambert, \& Andrade, 2003).

Wraparound is a system of care for children and families who require intensive mental health services. Many of the children receiving these services present with a variety of mental health problems including (but not limited to) autism, oppositional defiant disorder, ADHD, and various developmental delays. As a result, it is common that these children also display severe disruptive behavior problems (Burchard \& Clarke, 1991 as cited in Hyde, Burchard, \& Woodworth, 1996). Wraparound services include 
supports for the child and family involved in mental health, educational, juvenile justice, and child welfare systems to achieve positive outcomes. These services also include Therapeutic Staff Support (TSS), mobile support, and behavioral consultation intervention (Bugaj \& Manning, 2002). TSS, who were the focus of this study, are required to have a bachelor's degree and at least 1 year of experience in the field of mental health. In working with child clients between 3 and 20 hours per week, typically within the home, their primary role is to ensure that their client's treatment plan is being fulfilled. TSS may work with both the child client and his or her caregivers in order to meet treatment goals and obtain information regarding the child's problem behaviors.

To determine whether intensive services for children and adolescents are effective, more studies are needed that evaluate the outcomes of Wraparound by using strong methodological designs that include appropriate comparison groups (Suter \& Bruns, 2008). For instance, Stokes and McNeil (2014) conducted a study in which treatment outcomes of Wraparound were compared to the outcomes of Parent-ChildInteraction Therapy (PCIT; Eyberg, 1988), an evidence-based treatment for children ages 2-to-7 years with disruptive behavior problems. The Wraparound intervention was found to result in no significant improvements over a 7-month period, whereas PCIT demonstrated significant child behavior change (Stokes \& McNeil, 2014).

The findings from this study provided evidence that the core components of PCIT are effective even when implemented within a community-based setting. However, it should be noted that all therapists in both the PCIT and the usual care group (UC) of this study possessed advanced degrees, primarily Master's degrees. Furthermore, all therapists in the PCIT group had previously completed a 40-hour PCIT training within two years of the initiation of the study. Although it is a requirement that all therapists 
who provide PCIT services complete the 40-hour PCIT training, and possess at least a Master's degree, these circumstances are not always feasible within the realms of community-based services (PCIT International, 2013). Specifically, in the case of homebased services, many of the staff who implement services within the home are bachelor'slevel making them unable to deliver PCIT services. In an attempt to develop a method for bachelor's level staff to use the core components of PCIT to manage their client's disruptive behavior within the home, researchers developed Staff-Child Interaction Therapy, a protocol specifically developed for bachelor's-level home-based therapists. Staff-Child Interaction Therapy (SCIT) was developed to train adult mental health staff to manage behavior problems of children ages 2-to-9 years of age. The structure of SCIT was founded within the model of PCIT, and includes several elements of PCIT that have proven to be effective for child behavior management. These core components are based largely on the attachment theory (Bowlby 2005), which states that children are more likely to develop healthy attachments and relationships with their caregivers if they are able to trust that the caregiver will meet their basic needs. This theory shapes the foundation for the goals of Child-Directed Interaction (CDI), the first stage of SCIT (and PCIT), which are to create a warm and positive relationship between the staff member and their client. SCIT and PCIT are also largely founded within the coercion theory (Patterson, 1982), which explains the contingencies that maintain a child's behavior. Adult-Directed Interaction (ADI), the second stage of SCIT, is largely shaped by the coercion theory. During this stage, staff members learn to shape and modify the contingencies that maintain their client's negative behavior, and re-establish appropriate contingencies that maintain positive child behavior. SCIT also incorporates aspects of play therapy (Axline, 1969), which focuses on developing appropriate 
behavior through the use of nonverbal, but warm and playful interactions. Lastly, the two stages of SCIT (CDI and ADI) are based on Hanf's two-stage model of managing child behavior problems (Hanf, 1969). During the first stage of Hanf's two-stage model, caregivers are taught to only provide attention to their child's positive behaviors and to ignore negative attention-seeking behaviors. During the second phase, caregivers are taught to provide clear, firm directions, praise compliance, and provide time-out for noncompliance.

While SCIT incorporates many of the core treatment components of PCIT, the core training components used in PCIT are also utilized in SCIT. Behavioral skills training (BST) is one of the most common and effective approaches to teach individuals new skills (Hine, 2014), and comprises the majority of the methods used to train caregivers in PCIT. BST was also used in this study to train TSS. Behavioral skills training typically consists of instruction, modeling, rehearsal, and feedback. There have been several studies that have evaluated the effectiveness of using behavioral skills training to teach individuals new skills. Furthermore, several studies have demonstrated high success rates when behavioral skills training has been used to train staff, particularly staff working with children with developmental disabilities and disruptive behavior problems (Hine, 2014; Leblanc, Ricciardi, \& Luiselli, 2005; Nosik, Williams, Garrido, \& Lee, 2013). For instance, Sarkoff and Sturmey (2004) used a multiple baseline design to evaluate the use of behavioral skills training to train three teachers working with students with autism in discrete trial training. During baseline assessment, teachers performed 10 discrete trials with students. Then, teachers participated in an instruction phase in which researchers provided teachers with written instructions and reviewed them with the teachers. Next, teachers were provided a graph of their performance at baseline and given 
feedback from researchers. During the rehearsal phase, teachers performed three discrete trials to which researchers responded immediately with verbal feedback. Lastly, during the modeling phase, researchers demonstrated three discrete trials with the student and placed emphasis on components that the teacher performed incorrectly during the baseline assessment. The rehearsal and model phases were repeated. The teachers then participated in a post-training assessment during which they completed 10 uninterrupted discrete trials, for which they did not receive any instruction, feedback, modeling, or rehearsal practice. Results indicated that behavioral skills training contributed to a $50 \%$ increase in performance accuracy by teachers immediately following training. Overall, the use of behavioral skills training to train teachers and staff is supported with empirical evidence, and was therefore incorporated in this study to train staff in SCIT during the one-day workshop.

SCIT is based on the standard model of PCIT and there are several similarities between the two models, but there are also some noteworthy differences (see Appendix A for a comparison chart of SCIT and PCIT). First, standard PCIT typically involves coaching of both Child-Directed Interaction (CDI) and Parent-Directed Interaction (PDI) skills. During standard PCIT training, therapists learn how to coach caregivers on how to effectively implement both CDI and PDI skills during sessions with their client and the client's caregiver. However, SCIT-trained therapists will not be coaching caregivers during in-home sessions with their clients, instead they will be modeling these behavior management skills for caregivers. To deliver PCIT, it is required that one have at least a master's degree, 40 hours of face-to-face time with a certified PCIT trainer, and one year of training in the form of a workshop, co-therapist model, and/or online training (PCIT International, 2013). With only a bachelor's degree and case consultation, TSS are not 
qualified to coach PCIT; however, during the SCIT training, they are taught to model the skills, code caregivers' skills and provide feedback on progress towards mastery to the caregivers. Lastly, whereas PCIT is used primarily in outpatient mental health however, SCIT was developed to implement positive behavior management strategies in group homes, residential treatments, and crisis centers (Diamond, 2010).

In regard to the disciplinary phase, both models teach caregivers and staff to deliver effective commands, provide contingent praise, and provide consistent, predictable consequences for non-compliance; although, there are also differences between the two models concerning disciplinary sequences. During the PDI phase of standard PCIT, caregivers are taught to use a "time-out" chair and back-up room consequence in response to child noncompliance. However, in SCIT, this phase is known as Adult-Directed Interaction (ADI), and TSS are taught the "broken record" disciplinary technique in response to child noncompliance. Specifically, the adult (i.e., TSS or caregiver) implements the "broken-record technique," which involves repeating the command verbatim in a robotic tone for a maximum of three attempts. If the child still does not comply after the third command, the adult provides a warning statement that explains to the child that noncompliance will result in physical guidance to help the child execute the command. If the child still does not comply, the adult will place his/her hand over the child's hand and guide the child to complete the task. If the child still does not comply after a $5^{\text {th }}$ time, the adult will provide another warning statement regarding a restriction of privilege. Lastly, if the child does not comply, the adult explains that the child has lost a privilege (please see Appendix B for the standardized script used during ADI). 
The only publication on SCIT, a chapter in Parent-Child Interaction Therapy (Diamond, 2010), discusses how the first SCIT model was piloted at a child crisis center with children who were between the ages of 4 and 8 years. Treatment began with assessment of the needs of the child clients as well as observations of staff-child interactions between 4 staff members and 12 child residents using the Dyadic ParentChild Interaction System (DPICS; Eyberg, Nelson, Duke, \& Boggs, 2005). The interactions took place at an outdoor play area during a one-hour recess. The baseline assessment indicated that there was significant room for improvement for staff personnel who utilized numerous time-outs, displayed aggression, and used no labeled praise. Following the baseline assessment, SCIT was implemented through video demonstrations, didactic sessions, coaching, and feedback, procedures similar to that of PCIT. After the completion of SCIT training, the staff and child residents repeated the procedure used during the baseline data collection and were recorded interacting during a 1-hour recess. Results indicated that there was a decrease in time-outs as well as an increase in labeled praise during post-data collection. Staff also reported less stress, higher job satisfaction, and improved relations with the child clients. Although, this study demonstrated positive outcomes of the implementation of SCIT in the group setting, there were limitations that prevent it from providing sufficient empirical support for the effectiveness of SCIT: (a) there was no control group, meaning there was no way to compare outcomes of groups who completed SCIT to those outcomes of those who did not complete SCIT, or determine if any changes in behavior were actually due to the intervention (b) the study included a small sample size.

It should be noted that even though the intervention discussed in the Diamond (2010) study is referred to as "SCIT," as is the intervention in the current study, there are 
notable differences between the two. The study conducted by Diamond (2010) served as a pilot study of sorts in order to explore whether staff members who worked in community group centers could acquire CDI skills and increase the number of positive interactions with their students. However, the staff involved in this study did not learn any disciplinary strategies for managing disruptive behavior. This intervention shares greater similarities with Child-Adult Relationship Enhancement (CARE; Gurwitch, Messer, Masse, Olafson, Boat, \& Putnam, 2015), an intervention designed to enhance adult-child relationships, than it does with the version of SCIT discussed in this current study. CARE was initially developed as a prevention model for children who were at risk for child maltreatment, with the idea that positive caregiver-child relationships increase child compliance, thus also reducing risk for child maltreatment. This particular intervention was designed for implementation by any adult who interacts with children between the ages of 2 and 18. Both CARE and the version of SCIT discussed in the Diamond (2010) study, focused on improving caregiver relationships and making them more positive, however neither of these treatments involved disciplinary methods that addressed severe disruptive behavior. Increasing positive interactions between staff (TSS) and their child clients was one of the primary goals of this current study, however, TSS were taught several additional PCIT-based skills and disciplinary methods that have been shown to lead to effective child behavior management. The study involved the transfer of these skills from therapist to caregiver through modeling, coding, feedback, and masterybased skill acquisition. During the SCIT workshops, TSS learned and practiced effective ways to teach caregivers how to use SCIT skills during interactions with their own child. These components were not included in the Diamond (2010) study. 
SCIT was specifically developed for bachelor's-level home-based therapists (TSS) to assist them in managing the disruptive behavior of their young child clients between the ages of 2 and 9 years. The overall larger investigation is the first of its kind to evaluate an intervention for home-based bachelor's-level staff that is largely founded within the core skills and components of PCIT. The current study, however, was conducted as a pilot study and fidelity check to the overall larger outcome study. In other words, before an intervention can be evaluated, there must be methods in place to ensure that the intervention is implemented correctly and effectively with fidelity. A prerequisite for SCIT fidelity was the acquisition of new skills to a level of mastery that would enable the therapists to model, code, and provide feedback to caregivers in their homes. Thus, the purpose of the current study was to evaluate whether the training methods used could lead to skill acquisition for bachelor's-level therapists. Specifically, researchers developed a one-day training workshop that involved the use of behavioral skills training (BST) in order to evaluate it effectiveness in training the TSS in child behavior management skills. This investigation compared the effects of two separate workshops, SCIT versus an attention control (i.e., Compassion Fatigue) training workshop, on therapist skill change at a post-evaluation session that occurred 6-to-8 weeks after the initial workshop.

\section{Purpose of the Present Study}

In the current study, bachelor's-level personnel who provide intensive in-home services for child clients were trained in SCIT, a manualized approach to managing child problem behavior within the homes of their clients ( see Appendix C for SCIT skills taught to TSS workers). The current study was a part of a larger investigation evaluating 
the overall effectiveness of SCIT as a component of a home-based Wraparound service. The larger study evaluated SCIT outcomes by measuring changes in child problem behavior, staff sense of competence, and changes in relationship between staff and client. The present study serves as a fidelity check for the larger outcome investigation to determine the degree to which staff acquired the SCIT skills after completing the one-day SCIT workshop. The purpose of this particular study was to evaluate differences in skill change between TSS who received a SCIT workshop and TSS who received an attention control workshop (i.e., Compassion Fatigue) using behavioral coding of staff behavior during role-play situations.

\section{Study Hypotheses}

Research question. Did TSS who received a one-day SCIT training workshop demonstrate improvements in child behavioral management skills from baseline to posttraining assessment? Specifically, did TSS in the SCIT group display greater change on targeted skills while interacting with a confederate child (an undergraduate research assistant trained to behave like a young child) as compared to TSS who received a oneday attention control workshop from pre- to 6-to-8 weeks post-training?

a) It was expected that TSS who received the SCIT workshop and received feedback on their skills would have a greater increase on the positive skills composite from pretraining to 6-to-8 weeks post-training on the Dyadic Parent-Child Interaction Coding System (DPICS; Eyberg, Nelson, Duke, \& Boggs, 2005) as compared to TSS who received an attention control workshop. Specifically, TSS who were trained in SCIT were expected to display greater improvements on the positive skills of labeled praise, behavioral descriptions, and reflections than TSS in the attention control group during both the Child-Led Play and Clean-Up situations. 
b) It was expected that TSS who received the SCIT workshop and received feedback on their skills would have a greater decrease on the negative skills composite from pretraining to 6-to-8 weeks post-training on the DPICS (Eyberg, Nelson, Duke, \& Boggs, 2005) as compared to TSS who received an attention control workshop. Specifically, TSS who were trained in SCIT were expected to display a greater decrease in the negative relationship skills of questions, commands, and negative talk than TSS in the attention control group in both the Child-Led Play and Clean-Up situations.

c) It was expected that TSS who received the SCIT workshop and received feedback on their skills would display a greater increase in contingent labeled praise for child compliance from pre-training to 6-to-8 weeks post-training on the DPICS (Eyberg, Nelson, Duke, \& Boggs, 2005) as compared to TSS in the attention control group during the Clean-Up situation only.

d) It was expected that TSS workers who were trained in SCIT and received feedback on their skills would have a greater increase in effective commands from pretraining to 6-to-8 weeks post-training on the DPICS ( Eyberg, Nelson, Duke, \& Boggs, 2005) compared to TSS in the attention control group on the Clean-Up situation only.

\section{Method}

\section{Participants}

The participants in this study were TSS who were employed with Family Behavioral Resources (FBR), a community mental health agency located in Southern Pennsylvania $\left(M_{\text {years }}=4.48\right.$, range of years employed with FBR $=.42-18.5$ years, $S D=$ 4.9). There were a total of 39 TSS included in this study, 20 who received SCIT and 19 who received an attention control workshop. A total of $34(87.2 \%)$ TSS identified as 
female, while $5(12.8 \%)$ identified as male. The majority of the sample was Caucasian, as $35(89.7 \%)$ TSS identified as Caucasian, 2 (5.1\%) TSS identified as African American, and $2(5.1 \%)$ TSS identified as Other. Lastly, regarding level of education, 3 TSS $(2.6 \%)$ earned an Associates degree, 26 (66.7\%) TSS earned a Bachelor's degree, 8 (20.5\%) TSS possessed some graduate training, 1 (2.6\%) TSS earned a M.A./M.S. degree, and 1 (2.6\%) TSS earned a M.S.W./L.S.W degree. Therapists who did not attend both workshops at pre- and post- time points were excluded from the analyses ( $\operatorname{SCIT~} n=8$; attention control; $\mathrm{n}=4)$.

Research assistants attended staff meetings for the TSS in order to explain the study and answer the staff's questions. It was explained that participation in the study was completely voluntary, and the decision regarding participation would not affect their employment with Family Behavioral Resources. Consistent with the larger project from which this study is based, inclusion criteria for TSS were: (a) the child client had to be between 2 and 9 years of age, (b) the child client had to be able to comprehend basic commands, (c) the TSS had to have some contact with the caregivers of their child client, and (d) the child had to display disruptive behavior problems that were indicated by the TSS and corroborated by the Eyberg Child Behavior Inventory (ECBI; Eyberg \& Pincus) completed from the perspective of both the caregiver and TSS who worked with the child. Oppositional Defiant Disorder and Autism diagnoses were the two most common diagnoses of the child clients involved in the study. Please see Table 2 for percentages of each child diagnosis separated by sex of the child. TSS interested in taking part in the study and who worked with at least one family who met study eligibility were consented at the beginning of the workshop. 
TSS interested in this study were asked about their availability for specific workshop dates. To be included, TSS needed to be available for a pair of workshops; Day 1 was the pre-training assessment followed by a 5.5-hour workshop, while Day 2 occurred 6-to-8 weeks later and included a post-training assessment followed by an additional 6-hour workshop. TSS were not aware of whether they were signing up for a SCIT or an attention control (i.e., Compassion Fatigue) workshop. When TSS were available for only one pair of workshop dates, the TSS were assigned based solely on availability. When TSS were available for multiple workshop dates, they usually were assigned to a workshop condition based on the flip of a coin. However, there were occasional times when the TSS were available for both conditions, but were placed in a workshop due to researcher needs (e.g., insufficient sign-up for a particular day). Researchers made strong efforts to randomize, but logistical issues resulted in some therapists being assigned to groups based on availability alone. Across all assignments, however, TSS were not aware of which workshops were associated with each date, nor was the researcher aware of the skill level of each therapist. As a result, TSS did not have a choice about the content of the scheduled workshop. Due to the differences in training content between each workshop, TSS were asked to minimize the extent to which they discussed the trainings and their clients at the agency, specifically when TSS from a different workshop were present.

\section{Baseline Assessment}

TSS members assigned to both the SCIT and attention control conditions participated in the baseline assessment, which took place at the beginning of the workshops (immediately following informed consent procedures). As this study was conducted within a larger investigation of SCIT outcomes, TSS were asked to complete 
several measures used to evaluate outcomes of SCIT, including behavior change, change in staff sense of competence, and changes in relationship between staff and child client. Specifically, staff completed the following measures: a form regarding their demographics (TSS Demographic Form), Eyberg Child Behavior Inventory (Eyberg \& Pincus, 1999), Child Behavior Checklist (CBCL; Achenbach, 2001; Achenbach \& Rescorla, 2001) the Staff Sense of Competence Scale (SSOCS), a modified version of the Parenting Sense of Competence Scale (PSOC; Johnston \& Mash, 1989), Penn Therapist Facilitating Behaviors' Questionnaire Method (TFBQ; Alexander \& Luborsky, 1986), Additional Therapist Questionnaire (adapted from Bates \& Dozier, 1998; McCrae \& Costa, 1986; Pervin, 1990), and the Student Teacher Relationship Scale (STRS; Pianta, 2001), Knowledge of Behavioral Principles as Applied to Children (KBPAC; O’Dell, Tarler-Benlolo \& Flynn, 1979), a modified version of the Therapy Attitude Inventory (TAI; Brestan, Jacobs, Rayfield, \& Eyberg, 1999). However, with the exception of the TSS demographic form, these measures were included only in the larger study and were not analyzed for the current study.

To obtain a baseline measure of skill level, the TSS interacted with a confederate child during two structured DPICS situations (i.e., Child-Led Play and Clean-Up) while the research assistants observed and conducted live coding (see Appendix D for a copy of the coding sheet showing all coding categories; Eyberg, Nelson, Duke, \& Boggs, 2005). The DPICS includes a third observation, Parent-Led Play; however it was omitted from the study due to time constraints. The Clean-Up situation was chosen over the Parent-Led Play because it evokes commands and poses a common challenge during therapy sessions in the home. Only the DPICS observations and demographic information (TSS demographic form) were used in the current study. 


\section{Post-Training Assessment}

TSS in both study conditions completed post-training assessments immediately prior to the the second workshop, using the same procedures as the baseline assessment. The post-training assessment took place 6-to-8 weeks after the baseline assessment.

\section{Training and Coding of Observational Data}

TSS were pulled from the task of completing measures in the "measures room" to complete the DPICS assessment during both pre- and post-workshops. The TSS interacted with a confederate child, an undergraduate research assistant who was trained to behave like a young child client during two standard 5-minute DPICS assessment situations, Child-Led Play and Clean-Up, as well as during Child-Directed Interaction and Adult-Directed Interaction training practice that occurred during workshop breakout groups. The pre-and post-assessments lasted approximately 1 hour each depending on the number of participants and research staff. The evaluation was completed in small groups comprised of a TSS and two research assistants, one who played the role of a confederate child and one who served as a coder (i.e., coding the interactions between staff member and confederate child using the DPICS). The baseline and post assessments were completed using three separate rooms: a measures room for the completion of measures and two assessment rooms used for the actual DPICS assessment. While the majority of TSS completed measures in the measures room, individual TSS were pulled out to complete the DPICS assessment in one of the two assessment rooms, while the remaining TSS continued to work on their measures. The number of TSS who were pulled to complete an assessment at one time was dependent upon the number of research staff. Typically there were two groups ( 1 TSS, 1 coder, and 1 confederate child in each group) in each of the assessment rooms. On some occasions, however, when research staff was 
limited, or a small number of participants was present, there might have been only one group in each assessment room. One research assistant in each room read the DPICS instructions for each situation before the TSS interacted with the confederate child. Once they finished the initial DPICS assessment, TSS returned to the measures room to complete the remainder of their measures.

In addition to the research assistants who served as coders and confederate children within the breakout groups, an additional research assistant rotated around the room and served as a reliability coder. The reliability coder rotated among the breakout groups and switched groups after each DPICS situation. For instance, the reliability coder might have coded for reliability during Child-Led Play for the first breakout group and then rotated to another group to code for reliability during Clean-Up. When there was available staffing, two reliability coders were used. Percent agreement is the customary method for calculating interrater reliability for live observations using the DPICS (Eyberg, Nelson, Duke, Boggs, 2005). Interrater reliability (percent agreement) was calculated by dividing the total number of coder agreements by the total number of coder agreements plus disagreements.

Each interaction between the TSS and confederate child was live coded by research assistants who were trained to reliably code parent-child interactions using the DPICS. Video recording was not used, as adequate research staffing was sufficient to allow for live coding. All coders were trained using the The Abridged Workbook: Coder Training Manual for the Dyadic Parent-Child Interaction Coding System ( $4^{\text {th }}$ ed., Fernandez, Chase, Ingalls, \& Eyberg, 2006) and received approximately 20 hours of training. In addition, undergraduate coders were required to achieve a score of $80 \%$ or higher on at least $90 \%$ of the review quizzes, as well as reach $80 \%$ reliability with a 
graduate researcher on video coding. Reliability data were collected on $38 \%$ of the DPICS interactions (please see Table 1 for reliability percentages for each variable). Overall, reliability for individual dependent variables ranged from $80.9 \%$-to- $83.0 \%$. Undergraduate research assistants received approximately 5 hours of training to perform the role of confederate children. The confederate child training followed the procedure developed by Tempel, McNeil, Chengappa, and Costello (2014) in which PCIT-based parenting classes were provided in a women's correctional facility. A behavior checklist was used as a check to ensure that specific behaviors occurred consistently during interactions between the caregiver and confederate child. Research assistants who played the role of the confederate child referred to the list throughout the interactions. Specific behaviors that were on the checklist included tantrumming, screaming, singing, complying with commands, and throwing toys (see Appendix E for the Fidelity Checklist for Confederate Child Behavior during Role Play).

\section{Control Workshop}

TSS assigned to the attention control group completed a 6-hour workshop, addressing the topic of "Compassion Fatigue." Attention control group therapists also participated in exercises designed to generate discussion regarding the practices involved in their current home-based services. Following the baseline assessment, the first 1.5 hours of the workshop involved small breakout discussion groups during which the TSS were prompted to answer questions from 3 different categories: (a) day-to-day activities, (b) supervision, and (c) challenges in the home (see Appendix F for a list of discussion questions used during breakout groups). Each group consisted of 3-to-4 TSS, a graduate student researcher who led the discussion, and an undergraduate research assistant who was designated as a transcriber of all discussions. Following a one-hour lunch, one of the 
graduate student researchers provided a presentation on compassion fatigue prevention, the factors involved in burnout, and job-related stress. TSS were then shown a clip from a TV show which provided examples of job burnout and compassion fatigue. Following the presentation, TSS participated in a short game designed to facilitate discussion. Lastly, TSS were provided a bag of standardized SCIT toys to be used in-session with their child client (see Appendix G for the Control/Compassion Fatigue workshop protocol).

\section{SCIT Workshop}

SCIT training took place throughout an 6-hour-long workshop, and included the DPICS assessment, PowerPoint presentations, small exercises, coding of skills, workshop reflection. During the SCIT workshop, TSS received training in the DPICS, CDI skills, and ADI skills. Breakout sessions allowed TSS to role-play and practice each new skill. During breakouts, TSS practiced using newly acquired CDI and ADI skills as well as using the DPCIS for coding adult-child interactions. Research assistants (doctoral students and advanced undergraduate students who were trained in SCIT to the point of mastery) provided feedback for TSS regarding their skill use and coding. Several PowerPoint presentations were developed for the purpose of this study and used throughout the workshop (see Appendix C for the behaviors targeted in the SCIT workshop). The workshops typically ranged in size from 9-to-18 participants. The number of therapists per group was consistent with previous PCIT literature that used a group format and included a similar group size (e.g., Niec, Barnett, Prewett, \& ShanleyChatham, 2016; Nieter, Thornberry, \& Brestan-Knight, 2013).

Prior to the workshop, a graduate researcher explained informed consent. The next 55 minutes to 1 hour were spent on baseline assessment procedures. For the next 30 minutes, TSS were introduced to the workshop and trainers. Theory behind PCIT and 
logistics of using skills in the homes of the clients were explained. The next 45 minutes were spent explaining CDI skills, providing the rationale for using the PRIDE (i.e., Praise, Reflect, Imitate, Describe, Enjoy) skills, modeling, and teaching selective ignoring of negative attention-seeking behaviors. The next 30 minutes included training in the DPICS, which involved practice with coding live role-plays.

Following the DPICS training, TSS then spent the next hour practicing CDI skills and DPICS coding in the form of role-play in breakout groups of approximately 5 people, including 2 other TSS, 1 trained coder, and 1 confederate child. TSS alternated turns interacting with the confederate child and coding the TSS-confederate child interaction so that they had the opportunity to work towards mastery of both the PRIDE skills and the coding skills. The research assistants provided feedback and reliability checks on TSS performance after each role-play rotation. CDI practice was followed by a lunch break during which TSS were asked to complete one DPICS quiz.

Following lunch, for the next hour, TSS were taught how to use effective commands as well as identify and code child compliance. TSS were also provided with the rationale for Adult-Directed Interaction (ADI), and the discipline procedures used in SCIT were modeled by research staff. Specifically, TSS were taught to deliver effective commands followed by 5 seconds of silence, and to provide contingent praise for child compliance. In the case of noncompliance, TSS were also taught how to implement the "broken record" technique (see Appendix B for the standardized script involved in ADI). Following a 15-minute break, TSS spent the next hour practicing the use and coding of ADI skills during breakout role plays of approximately 5 people, including 2 other TSS, 1 coder, and 1 confederate child. TSS alternated interacting with the confederate child and coding TSS-confederate child interactions so that they had the opportunity to practice and 
code ADI skills. The final 40 minutes of the workshop included TSS reflections about the training, as well as a 15-minute review about how TSS should conduct a typical SCIT inhome session. TSS were also asked to complete a satisfaction survey about the workshop. Lastly, TSS were provided a bag of standardized SCIT toys to be used in-session with their child client (please see Appendix H for the SCIT workshop protocol).

\section{Mastery Criteria}

TSS in the SCIT group were encouraged to strive towards mastery in each of the following training areas throughout the workshop: CDI, ADI, coding reliability, and $80 \%$ accuracy on one DPICS quiz. TSS had to meet the mastery criteria set for each training area in order to be considered competent in SCIT (please see Appendix I for mastery criteria for each of the training areas). TSS had an opportunity to meet mastery for CDI, ADI, and coding reliability during role-play within break-out groups, while quizzes were taken during lunch. Upon meeting mastery criteria for a specific training area, TSS were given a colored star that corresponded with the mastery criteria for that specific training area. The stars were placed on the back of the ID tags of the TSS, as a method to track progression towards mastery in all areas. During the workshop, $67 \%$ of staff met mastery criteria of CDI, $77 \%$ of staff met mastery criteria of ADI, $77 \%$ of staff met mastery criteria of CDI DPICS Reliability, and $87 \%$ of staff met mastery criteria of DPICS scoring ( $80 \%$ or higher on a DPICS quiz).

\section{Measures}

TSS Demographic Form. At the baseline assessment, the TSS were asked to complete a demographic form that inquired about the following information: therapist sex, therapist race/ethnicity, years in current position, highest degree earned, specific training received, familiarity with PCIT, amount of time TSS had worked with client, 
number of hours per week TSS member spent with client, past and current client involvement with child protective services, and client mental disorder diagnoses (see Appendix $\mathrm{J}$ for the TSS Demographic Form).

Dyadic Parent-Child Interaction Coding System. TSS interactions with the confederate child were coded using the Dyadic Parent-Child Interaction Coding System III (DPICS; Eyberg, Nelson, Duke, \& Boggs, 2005) at the baseline and post-training assessment. DPICS provides a method of observing and analyzing social interactions that occur between a child and caregiver. The DPICS was designed to provide a consistent method of coding parent-child interactions, child behavior problems, and parenting skills, and is also commonly used to code staff-and teacher-child interactions. The DPICS observations in this study occurred during the two DPICS situations: Child-Led Play and Clean-Up. The situations each lasted for 5 minutes for a total of 10 minutes of observations (refer to Appendix $\mathrm{K}$ for the variables that were coded during each situation, as well as definitions of each of those variables; refer to Appendix L for the script that was used by the researchers to instruct the TSS to transition from one situation to the next.

The DPICS has been shown to have both adequate reliability and validity across its categories including real-time and video recorded observations (Bessmer, 1998; Deskins, 2005; Foote, 2000; Robinson \& Eyberg, 1981). In a study conducted by Lyon and Budd (2010), in which PCIT was implemented in a community mental health setting amongst low SES families, the DPICS demonstrated reliability across all categories included. Specifically, kappa values ranged from moderate (.61) to high (.88). Additionally, a study conducted by Bagner and Eyberg (2007) in which 
researchers conducted a randomized controlled trial in attempts to explore the effectiveness of PCIT in reducing behavior problems among children with developmental delays, live coding was used to assess parent-child interactions on each of the individual DPICS categories. Values for percent agreement ranged from $67 \%$ - to-91\%, with reflections resulting in the lowest percent agreement and information questions resulting in the highest. Lastly, in a study conducted by Robinson and Eyberg (1981), typical families were compared to families with children who displayed conduct problems in order to assess for validity. It was found that the DPICS correctly classified $100 \%$ of normal families, $85 \%$ of treatment families, and $94 \%$ of all families (Robinson \& Eyberg, 1981). The DPICS also has been shown to be sensitive to parenting skill changes associated with Parent-Child Interaction Therapy (e.g., Bjørseth, McNeil, \& Wichstrøm, 2015).

\section{Power Analysis}

Power analyses were conducted with G*Power (Faul, Erdefelder, Lang, \& Buchner, 2007) for sample size estimation. With an alpha of .05 and power at the level of 0.80, it was determined that 40 participants would be sufficient to detect a large effect using a repeated measures ANOVA with between (Group) and within (Time) effects. Furthermore, 40 participants was a feasible number given the the number of research staff available. Prior evaluations of PCIT and TCIT consistently have demonstrated effects in the medium to large range (e.g., Thomas \& Zimmer-Gembeck, 2007).

\section{Interrater Reliability Assessment}

Interrater agreement between the primary and reliability coder was evaluated. Agreement was calculated by dividing the number of agrees by the sum of agrees and disagrees. The overall average interrater reliability for all categories combined including 
both pre-and post-training was $82.11 \%$. Additionally, reliability was evaluated for each of the following dependent variables separately: positive composite, negative composite, contingent praise, and effective commands (see Table 1 for the average interrater reliability and ranges for each of the dependent variables).

\section{Results}

\section{Preliminary Analyses}

Preliminary analyses were conducted to check for violations of assumptions. These analyses indicated that that there were violations of assumptions of normality, linearity, and/or homoscedasticity for the following variables: post positive composite, post negative composite, effective commands, and contingent praise. Data transformations were performed in attempts to correct violations; specifically, square root, $\log$, and inverse transformations were performed. Each variable was positively skewed, thus making it appropriate to use these transformations. Square root transformations were performed first, followed by log and inverse transformations. However, these transformations did not correct for violations of assumptions. Specifically, inverse transformations slightly improved normality, but worsened homogeneity for the post positive composite, post negative composite, effective commands, and contingent praise variables. Thus, the transformations were not used.

Descriptive data, consisting of the means and standard deviations for all independent, dependent, and demographic variables of interest, were summarized. The TSS Demographic Form provided descriptive data for overall characteristics of both groups. TSS demographic variables included (a) how long the TSS has worked with the identified client, and (b) hours spent with the client each week. Child demographic variables of interest included (a) age, (b) sex, (c) mental disorder 
diagnosis, (d) how long the child has received mental health services, and (e) past and current involvement with child protective services. Chi-square analyses were conducted to examine if groups differed on any categorical demographic variables (see Table 2). Independent samples t-tests were conducted to examine if SCIT and attention control group TSS differed on any continuous demographic variables at the baseline assessment (see Table 3). Finally, independent samples t-tests were conducted to examine if TSS in the SCIT and attention control groups differed on any dependent variables at the baseline assessment (see Tables 4 and 5). Chi square and t-test analyses indicated that there were no significant differences on any of the demographic or dependent variables between groups at baseline; thus no covariates were included in the primary analyses.

\section{Results for Therapist Positive Composite}

Analyses indicated that the therapists in the SCIT group displayed a significantly greater increase in positive composite skills from pre-training to 6-to-8 weeks post-training on the DPICS (Eyberg, Nelson, Duke, \& Boggs, 2005) than therapists in the attention control group for the Child-Led Play situation (see Table 6), but not for the Clean-Up situation (see Table 7). A 2 (Time [pre, post]) x 2 (Group [SCIT, Compassion Fatigue]) ANOVA indicated a significant Time x Group interaction, $\mathrm{F}(1,37)=.49 .68, \mathrm{p}<.05$, such that therapists in the SCIT group gave significantly more positive verbalizations (i.e., labeled praises, reflections, and behavior descriptions), than the therapists in the attention control group from pre-to 6-to-8 weeks post-training in Child-Led Play (see Table 6). The SCIT group displayed an average increase of 17.26 positive skills (labeled praises, reflections, and behavior descriptions), while the TSS in the attention control group displayed an 
average increase of 1 positive skill. See Figure 1 for a graph of these results. A post hoc Tukey test indicated that there were significant differences between the SCIT group at pre and post-assessment at $\mathrm{p}<.05$; the SCIT group at post was also significantly different than the attention control group at post at $\mathrm{p}<.05$. However, during the Clean-Up situation, the SCIT group did not display significantly greater change than attention control group on positive composite variable from pre- to 6-to8 weeks post- training $\mathrm{F}(1,37)=1.932, \mathrm{p}=.173$ (see Table 7 ).

\section{Results for Therapist Negative Composite}

Analyses indicated that the therapists in the SCIT group displayed a significantly greater decrease from pre-to 6-to-8 weeks post-training than therapists in the attention control group on the negative composite skills for both Child-Led Play and Clean-Up situations over time (see Tables 6 and 7). A 2 (Time [pre, post]) $\mathrm{x}$ 2 (Group [SCIT, Compassion Fatigue]) ANOVA indicated a significant Time $\mathrm{x}$ Group interaction, such that therapists in the SCIT group used significantly fewer commands, criticisms, and questions than did the therapists in the attention control group during the Child-Led Play situation from pre-to 6-to-8 weeks post-training $\mathrm{F}(1,37=10.930), \mathrm{p}<.05$. TSS in the SCIT group demonstrated an average decrease of 18.95 negative skills (questions, commands, and negative talk), while the TSS in the attention control group demonstrated an average decrease of 7.95 negative skills. See Figure 2 for a graph of these results. A post hoc Tukey test indicated that there were significant differences between the SCIT group at pre and post-assessment at $\mathrm{p}$ $<.05$; the SCIT group at post was also significantly different than the attention control group at post at $\mathrm{p}<.05$. With respect to the Clean-Up situation, commands were not included in the negative composite as they were required for the task. A 2 
(Time [pre, post]) x 2 (Group [SCIT, Compassion Fatigue]) ANOVA with repeated measures indicated a significant Time $\mathrm{x}$ Group interaction, such that therapists in the SCIT group issued fewer critical statements and asked fewer questions than did the therapists in the attention control group during the Clean-Up situation $\mathrm{F}(1,37)=$ $5.99, \mathrm{p}<.05$. TSS in the SCIT group demonstrated an average decrease of 9.89 negative skills (questions and negative talk), while the TSS in the attention control group demonstrated an average decrease of 3.35 negative skills. See Figure 3 for a graph of these results.

\section{Results for Effective Commands}

Percentage of effective commands was only assessed during the Clean-Up situation, as therapists were instructed to let the child lead the play during Child-Led Play; thus if commands were given during Child-Led Play, they were factored into the negative composite score. Analyses indicated that there was no significantly greater increase between the therapists in the SCIT group and the therapists in the attention control group on percentage of effective commands from pre-training to 6to-8 weeks post-training (Table 7). A 2 (Time [pre, post]) x 2 (Group [SCIT, Compassion Fatigue]) ANOVA indicated that the SCIT therapists did not give significantly more effective commands than did the therapists in the attention control group from pre-training to 6-to- 8 weeks post-training $\mathrm{F}(1,37)=0.978, \mathrm{p}=.329$ (see Table 7).

\section{Results for Contingent Praise}

Contingent praise was only assessed during the Clean-Up situation, as it only follows child compliance to a command and therapists were instructed to let the child lead the play during Child-Led Play. Analyses indicated that there was no 
significantly greater increase among therapists in the SCIT group and therapists in the attention control group on the contingent praise variable from pre-training to 6to-8 weeks post-training (Table 7). A 2 (Time [pre, post]) x 2 (Group [SCIT, Compassion Fatigue]) ANOVA indicated that the SCIT therapists did not give significantly more contingent praise following compliance than did the therapists in the attention control group from pre-to post-training, $\mathrm{F}(1,37)=5.371, \mathrm{p}=.090($ see Table 7).

\section{Discussion}

The purpose of this study was to evaluate the methods used to train TSS in SCIT, a newly developed protocol designed to assist staff in managing disruptive child behavior problems. While the goal of the overall larger study was to evaluate the effectiveness of the outcomes of the actual intervention, the current study served as a fidelity check to ensure that the workshops were effective in teaching TSS new skills, a crucial component to accurately evaluating SCIT. Specifically, this study examined the effectiveness of a one-day workshop for training therapists in StaffChild Interaction Therapy (SCIT), a newly developed protocol designed to assist bachelor's-level mental health staff in managing child problem behavior. The study explored whether the therapists displayed an increase in (a) positive verbalizations (labeled praise, reflection, behavior description), (b) effective commands, and (c) contingent praises, as well as a decrease in negative verbalizations (negative talk, questions, and commands), as a result of completing the one-day SCIT training. Outcomes were assessed during a one-hour assessment at the beginning of both the pre-treatment and post-treatment workshops, during which therapists interacted with confederate children while researchers coded the interactions using the DPICS 
(Eyberg, Nelson, Duke, \& Boggs, 2005).

It was found that the SCIT training workshop had a significant effect on the therapist positive composite score during the Child-Led Play situation and the therapist negative composite score during both the Child-Led Play and Clean-Up situations. In other words, therapists in the SCIT group gave more labeled praises, behavior descriptions, and reflections during the Child-Led Play situation, but not the Clean-Up situation, as compared to therapists in the attention control group from preto post-training. Furthermore, during Child-Led Play and Clean-Up situations, therapists trained in SCIT (a) provided fewer questions, critical statements, and commands, and (b) used fewer questions and negative verbalizations as compared to therapists in the attention control group at the post-training assessment. TSS in the control group displayed small changes in negative composite variables across the Clean-Up situations however, the changes were minimal in comparison with TSS in the SCIT group. Analyses concluded there was an effect size of 0.51 (Cohen's D) for TSS in the control group, and an effect size of 1.48 (Cohen's D) for TSS in the SCIT group. Considering the TSS in the control group did not receive any SCIT training, it would seem that these changes might have occurred as a result of the maturation effect. In other words, it is possible that TSS naturally become more skilled in child behavior management over time as they begin to adapt and build stronger rapport with their child client.

Although the findings on Child-Directed skills generally supported the efficacy of the SCIT workshop, the results with respect to Adult-Directed skills (e.g., effective commands, and contingent praise for compliance) were less promising. One possible explanation is that the amount of training provided to the therapists was 
not sufficient in order for major effects to be seen across all variables. Therapists received about 4.5 hours of interactive and didactic training, as the remainder of the day involved assessment, informed consent, lunch break, and workshop evaluation. In considering the components of standard PCIT training for Master's level therapists, the SCIT training was significantly less comprehensive. For PCIT certification, therapists are required to complete 40 hours of face-to-face training, consultation via phone or in-person at least twice per month for a year (i.e., 24 hours of phone consultation), video review of at least 4 PCIT sessions with written feedback from a PCIT trainer or session observation from a certified trainer, and experience with PCIT cases under consultation of a certified trainer (PCIT International, 2011). In contrast, this SCIT training included only 4.5 hours of faceto-face training and no consultation. This also proved to be a limitation to the overall study.

Furthermore, therapists were told to continue using their previously designed treatment plan with their clients until they completed a second workshop day 6-to-8 weeks after the first workshop. Thus, the therapists in this study received less than 5 hours of training and minimal practice post workshop, as compared to therapists trained via standard PCIT training who receive at least 40 hours of live training, 24 hours of consultation, and the opportunity to improve their skills by seeing clients.

However, the positive results found in the area of Child-Directed skills support the training format used in this investigation. The SCIT workshop involved behavioral techniques founded in learning theory that have been shown to be effective for teaching new skills to parents, teachers, and staff (Bennett-Levy, McManus, Westling, \& Fennell, 2009; Hine, 2014; Serketich \& Dumas, 1996). The core techniques of behavioral skills 
training, role-play, rehearsal, coding, and feedback, were used in the workshop. A positive outcome of this study was that only 4.5 hours of behavioral skills training led to $55 \%$ of the SCIT therapists reaching mastery by the end of the workshop day, and several changes in therapist skill that were maintained 2 months later. Yet, the lack of findings in two of the compliance training skills (effective commands and contingent praise) suggests that additional training is needed to obtain improvements in all of the complex skills needed to be an effective SCIT therapist.

A notable strength of this investigation is the use of behavioral coding to evaluate the workshop. Most studies supporting the effectiveness of a one-day training workshop have examined some form of change in perception or knowledge, rather than actual skill (Boulton, 2014; Humphries \& Nolan, 2015). For example, Boulton (2014) evaluated cognitive-behavioral training workshops for teachers by assessing self-efficacy beliefs, perceived effectiveness, and use of cognitivebehavioral interventions before and after either a three-day or one-day training workshop. In this particular study, the amount of cognitive-behavioral intervention was assessed, but new development of the teachers' skill level in implementing the interventions themselves was not. Additionally, it was shown that the three-day training workshop resulted in more positive results than the one-day training workshop. Furthermore, many workshop evaluation studies assess for changes in outcomes only at the immediate conclusion of the workshop day; however, this particular study assessed skill outcomes after 6-to-8 weeks. Thus, it is possible that some dependent variables would have been statistically significant had outcomes been assessed at the end of the SCIT training workshop rather than the 6-to- 8 weeks post-training assessment. Nevertheless, the results do support the effectiveness of a 
one-day SCIT workshop to teach bachelor's-level therapists certain child behavior management skills that can be successfully maintained over the course of approximately 2 months.

There were several limitations associated with this study that may have compromised the generalizability of the findings. First, the assessment of therapist skill change relied solely on role-play situations between the therapists and confederate children in a controlled classroom setting. Given the analogue nature of the observations, it is difficult to ensure the validity of the skill assessment as it relates to therapist-child interactions in the client's home environment.

In addition, due to limited staff and higher attendance at some workshops than others, interrater reliability for the behavioral observations was assessed unequally throughout the study. For instance, during some workshops, every assessment group was coded for reliability, while other workshops involved reliability measurements for only a subset of the training groups. Furthermore, interrater reliability was low on some variables, particularly for cases in which the frequency of a particular skill was low. For example, if a TSS only provided 3 labeled praises during the assessment, and 1 research coder marked 3 labeled praises, while the reliability coder marked 2 labeled praises, the reliability percentage would only be $67 \%$. Low frequency of occurrence was the case for all variables with reliability percentages that were $50 \%$ and lower. Other researchers have had similar problems with obtaining adequate interrater reliability on low frequency DPICS behaviors. For example, the percent agreement in this investigation was comparable to interrater reliability values found in the study conducted by Bagner and Eyberg (2007). Further regarding reliability, researchers did not calculate kappa values due 
to restraints on the number of research staff.

The rate of attrition was another limitation of the study, as some participants did not return to the post-assessment, thereby decreasing the sample size of the study. There were two primary reasons for attrition between the two groups. Firstly, employee turnover within Family Behavioral Resources (FBR) was a large contributor to attrition across both the SCIT group and the attention control group. In other words, there were several TSS who left FBR for various reasons (e.g. change in location, pursuit of higher education) over the duration of the study, and as a result, did not return to the second workshop. Secondly, client drop-out also contributed to attrition across both groups. For the majority of these cases, if the client discontinued services with FBR, the TSS no longer had a client who met inclusion criteria for the larger study; thus, that TSS did not return to the second workshop. The small sample size reduced the power of the study resulting in a decreased ability to obtain statistical significance on variables demonstrating a low to medium effect size. The attrition also raised the potential confound of a possible sample bias with the completer group being different from the initial sample.

Another limitation of the study involved violations to the assumptions of ANOVA (e.g. homoscedasticity, normality). Although appropriate data transformations were performed, they still did not fully correct for these statistical violations. While the lack of normality in the data confounded the interpretation of the findings, ANOVA is considered relatively robust with respect to violations of normality (Glass, Peckham, \& Sanders, 1972; Vasey \& Thayer, 1987). Lastly, although randomization procedures were employed when possible, randomization was not maintained throughout the study. Due to conflicts between therapists' 
schedules and specific workshop dates, researchers often had to forego randomization and assign therapists to either the treatment or attention control groups based on their availability to attend the sequence of three workshops. Yet, to prevent bias in group assignment, therapists with scheduling limitations remained unaware of the workshop content associated with the various sign-up dates.

Regarding TSS interaction between groups, even though efforts were made to ensure that there was no contamination between the SCIT group and the attentioncontrol group, this might have been a possible limitation that could not be fully avoided. Being that the TSS from both the SCIT group and the attention control group were employed at the same agency, it was possible that there would be some interaction between the two groups. However, it appeared that TSS from the two groups seldom worked together, as their home-based work did not require them to have designated office space. The majority of the TSS reported obtaining individual, rather than group supervision, thereby limiting the opportunities for TSS from the two different groups to communicate with each other about clients. Furthermore, researchers requested that the TSS attempt to avoid talking about the training around other TSS and while at the agency. Researchers also requested that the supervisors further emphasize the point to their supervisees.

Lastly, this study involved only bachelor's-level therapists from one particular agency in Pennsylvania. Although these therapists were recruited from multiple sites within the agency, this limits the study's generalizability to other therapists not employed with the agency. It is possible that FBR employs therapists with specific characteristics that could have affected the level of skill acquisition during the workshop, thus impacting the outcomes of the study. In the future, it 
would be beneficial to recruit therapists from several types of mental health agencies.

An important future direction based on this study is to evaluate skill change after therapists complete two SCIT training workshops as opposed to just the single training workshop. Findings indicating better results on Child-Directed Interaction than Adult-Directed Interaction suggest that Adult-Directed Interaction may be more challenging and require a greater amount of workshop time than allotted in the current investigation. As SCIT involves two distinct sets of skills for Child-Directed Interaction and Adult-Directed Interaction, learning could potentially be enhanced by allowing mastery and practice of one set of skills before introducing a new skill set. In addition, it would be optimal for the therapists' skill to be measured through interactions with their child client in a natural environment as opposed to measuring therapists' skill through interactions only during analogue role plays. Finally, future studies should take into account the high rate of turnover in both home-based therapists and home-based clients. Future investigations should plan on recruiting more participants than the current study, to allow for attrition but still maintain adequate statistical power to detect small to medium changes in therapist behaviors.

Overall, the goal of this study was to evaluate the effectiveness of a one-day workshop used to train bachelor's-level therapists in SCIT, a newly developed protocol for bachelor's-level therapists to deliver services to child clients in-home. The findings indicated that the one-day SCIT workshop was successful in not only teaching bachelor's-level therapists to use effective skills to manage child problem behavior, as half of the therapists mastered all 4 training areas by the end of the workshop, but many of these skills appeared to be maintained 6-to-8 weeks later at 
the DPICS post-assessment. Thus, SCIT workshops may be an effective method to train bachelor's-level therapists who deliver services to children in the home. In addition, the SCIT model is a promising approach to disseminating PCIT-based skills to bachelor's-level therapists who do not have the training or credentials to provide PCIT. In that way, SCIT training workshops could result in an improvement in home-based mental health services. Overall, the current study provided preliminary evidence for the effectiveness of a SCIT workshop approach for improving therapist skills. Future studies should investigate whether immersing SCIT within the current Wraparound model may increase the quality of community and home-based services delivered to children with behavioral and emotional challenges. 


\section{References}

Achenbach, T. M., \& Rescorla, L. (2001). ASEBA school-age forms \& profiles. Burlington: Aseba.

Achenbach, T. M., \& Rescorla, L. (2001). ASEBA school-age forms \& profiles. Burlington, VT: Aseba.

Alexander, L. B., \& Luborsky, L. (1986). The Penn Helping Alliance Scales. In L. S. Greenberg \& W.M. Pinsoff (Eds.), The psychotherapeutic process: A research handbook. (pp. 325366). New York: Guilford Press.

Bagner, D. M., \& Eyberg, S. M. (2007). Parent-child interaction therapy for disruptive behavior in children with mental retardation: A randomized controlled trial. Journal of Clinical Child and Adolescent Psychology, 36(3), 418-429.

Bates, B., \& Dozier, M. (1998). This is My Baby" coding manual. Unpublished manuscript, University of Delaware, Newark.

Bennett-Levy, J., McManus, F., Westling, B. E., \& Fennell, M. (2009). Acquiring and refining CBT skills and competencies: Which training methods are perceived to be most effective?. Behavioural and Cognitive Psychotherapy, 37(05), 571-583.

Bessmer, J. L. (1998). The Dyadic Parent-Child Interaction Coding System II (DPICS II): Reliability and validity. Dissertation Abstracts International, 58, 3961.

Bickman, L., Smith, C. M., Lambert, E. W., \& Andrade, A. R. (2003). Evaluation of a congressionally mandated wraparound demonstration. Journal of Child and Family Studies, 12(2), 135-156. 
Bjørseth, Å., McNeil, C., \& Wichstrøm, L. (2015). Screening for behavioral disorders with the Dyadic Parent-Child Interaction Coding System: Sensitivity, specificity, and core discriminative components. Child \& Family Behavior Therapy, 37(1), 20-37. doi:10.1080/07317107.2015.1000228

Boulton, M. J. (2014). Teachers' Self-Efficacy, Perceived Effectiveness Beliefs, and Reported use of cognitive-behavioral approaches to bullying among pupils: effects of in-service training with the I DECIDE program. Behavior Therapy, 45(3), 328-343.

Brestan, E., Jacobs, J., Rayfield, A., \& Eyberg, S.M. (1999). A consumer satisfaction measure for parent-child treatments and its relationship to measures of child behavior change. Behavior Therapy, 30, 17-30.

Bugaj, S. J., \& Manning, R. L. (2002). Suggestions for Improving the Delivery of Therapeutic Staff Support in the Public Schools. Journal of Mental Health Counseling, 24(1), 88-93.

Burchard, J. D., \& Clarke, R. T. (1990). The role of individualized care in a service delivery system for children and adolescents with severely maladjusted behavior. Journal Of Mental Health Administration, 17(1), 48-60.

Deskins, M. M. (2005). The Dyadic Parent-Child Interaction Coding System II (DPICS II): Reliability and validity with school aged children. Dissertation Abstracts International, 66, 2302.

Diamond, G. (2010). Staff- Child Interaction Therapy. In McNeil, C. B., \& Hembree-Kigin, T. L. (2010). Parent-Child Interaction Therapy: Second Edtion. NY: Springer

Eyberg, S.M. (1988). Parent-child interaction therapy: Integration of traditional and behavioral concerns. Child \& Family Behavior Therapy, 10, 33-46.

Eyberg, S. M., Nelson, M. M., Duke, M., \& Boggs, S. R. (2005). Manual for the dyadic parentchild interaction coding system. Unpublished manuscript. 
Eyberg, S. M., Nelson, M. M., Ginn, N. C., Bhuiyan, N., \& Boggs, S. R. (2013). Dyadic parent child interaction coding system: Comprehensive manual for research and training.

Eyberg, S. M., \& Pincus, D. (1999). Eyberg Child Behavior Inventory and Sutter-Eyberg Student Behavior Inventory-Revised: Professional manual. Psychological Assessment Resources.

Eyberg, S. M., \& Robinson, E. A. (1981). Dyadic parent-child interaction coding system. Seattle, WA: Parenting Clinic, University of Washington.

Farmer, E. M., Dorsey, S., \& Mustillo, S. A. (2004). Intensive home and community interventions. Child and Adolescent Psychiatric Clinics of North America, 13(4), 857884.

Faul, F.E., Lang, A.G., \& Buchner, A. (2007). G*Power 3: A flexible statistical power analysis program for the social, behavioral, and biomedical sciences. Behavior Research Methods, $39,175-191$.

Fernandez, M. A., Chase, R. M., Ingalls, C. A., \& Eyberg, S. M. (2005). The workbook: Coder training manual for the Dyadic Parent-Child Interaction Coding System. Unpublished manual. Retrieved August, 15, 2007.

Foote, R. C. (2000, March). The Dyadic Parent-Child Interaction Coding System II (DPICS II): Reliability and validity with father-child dyads. Dissertation Abstracts International, 60, 4886.

Glass, G. V., Peckham, P. D., \& Sanders, J. R. (1972). Consequences of failure to meet assumptions underlying the fixed effects analyses of variance and covariance. Review of Educational Research, 237-288.

Gurwitch, R. H., Messer, E. P., Masse, J., Olafson, E., Boat, B. W., \& Putnam, F. W. (in press). Child-Adult Relationship Enhancement (CARE): An evidence-informed program for children with a history of trauma and other behavioral challenges. Child abuse \& neglect. 
Hine, K. M. (2014). Effects of behavioral skills training with directed data collection on the acquisition of behavioral practices by workers in a private, not-for-profit child care center. Journal of Organizational Behavior Management, 34(3), 223-232.

Humphries, H., \& Nolan, M. (2015). Evaluation of a brief intervention to assist health visitors and community practitioners to engage with fathers as part of the healthy child initiative. Primary Health Care Research \& Development, 4, 1-10.

Johnston, C., \& Mash, E.J. (1989). A measure of parenting satisfaction and efficacy. Journal of Clinical Child Psychology, 18, 167-175.

Leblanc, M. P., Ricciardi, J. N., \& Luiselli, J. K. (2005). Improving discrete trial instruction by paraprofessional staff through an abbreviated performance feedback intervention. Education and Treatment of Children, 28(1), 76-82.

Lyon, A. R., \& Budd, K. S. (2010). A community mental health implementation of Parent-Child Interaction Therapy (PCIT). Journal of Child And Family Studies, 19(5), 654-668. doi:10.1007/s10826-010-9353-z

McCrae, R. R., \& Costa, P. T., Jr. (1986). A five-factor theory of personality. In L. A. Pervin \& O. P. John (Eds.), Handbook of Personality: Theory and Research ( $2^{\text {nd }}$ ed.). New York: Guilford.

McNeil, C. B., \& Hembree-Kigin, T. L. (2010). Parent-Child Interaction Therapy. Second Edition. New York: Springer.

National Institute for Health Care Management Research and Educational Foundation (2005). Health care quality research. Retrieved from http:// http://www.nihcm.org/index.php.

Niec, L. N., Barnett, M. L., Prewett, M. S., \& Shanley, C. J. (2016). Group Parent-Child Interaction Therapy: A Randomized Control Trial for the Treatment of Conduct Problems in Young Children. Journal of consulting and clinical psychology. 
Nieter, L., Thornberry Jr, T., \& Brestan-Knight, E. (2013). The effectiveness of group parentchild interaction therapy with community families. Journal of Child and Family Studies, 22(4), 490-501.

Nosik, M. R., Williams, W. L., Garrido, N., \& Lee, S. (2013). Comparison of computer based instruction to behavior skills training for teaching staff implementation of discrete-trial instruction with an adult with autism. Research in developmental disabilities, 34(1), 461468.

O'Dell, S. L., Tarler-Benlolo, L., \& Flynn, J. M. (1979). An instrument to measure knowledge of behavioral principles as applied to children. Journal of Behavior Therapy and Experimental Psychiatry, 10(1), 29-34.

PCIT therapist requirements. (2013, March 28). Retrieved from http:// http://www.pcit.org/therapist-requirements.html

Pennsylvania Department of Welfare (2001). Guidelines for best practice in child and adolescent mental health services. Retrieved from http://www.dhs.pa.gov/cs/groups/public/documents/manual/s_001583.pdf

Pervin, L. A. (1990) Handbook of personality: Theory and Research. New York: Guilford Press.

Pianta, R. C. (2001). Student-Teacher Relationship Scale: Professional manual. Psychological Assessment Resources, Lutz, FL .

Robinson, E. A., \& Eyberg, S. M. (1981). The dyadic parent-child interaction coding system: Standardization and validation. Journal of Consulting and Clinical Psychology, 49(2), 245.

Sarokoff, R. A., \& Sturmey, P. (2004). The effects of behavioral skills training on staff implementation of discrete-trial teaching. Journal of Applied Behavior Analysis, 37(4), 535-538. 
Serketich, W. J., \& Dumas, J. E. (1996). The effectiveness of behavioral parent training to modify antisocial behavior in children: A meta-analysis. Behavior Therapy, 27(2), 171186.

Stokes, J. \& McNeil, C.B. (2014). Effectiveness of Community-Delivered Parent-Child Interaction Therapy Compared to Treatment as Usual (Unpublished doctoral dissertation). West Virginia University.

Tempel A. T., McNeil, C. B., Chengappa, K., \& Costello, A. H. (2014). Evaluation of an existing parenting class within a women's state correctional facility and a parenting class modeled from Parent-Child Interaction Therapy. Children and Youth Services Review, 46, 238247.

Thomas, R., \& Zimmer-Gembeck, M. J. (2011). Accumulating evidence for parent-child interaction therapy in the prevention of child maltreatment. Child Development, 82(1), 177-192.

Vasey, M. W., \& Thayer, J. F. (1987). The continuing problem of false positives in repeated measures ANOVA in psychophysiology: A multivariate solution. Psychophysiology, 24(4), 479-486. 
Table 1

Reliability Percentages for Dependent Variables Pre-and Post-training

\begin{tabular}{ccccc}
\hline & Pre $^{\mathrm{a}}$ & Post $^{\mathrm{a}}$ & Overall $^{\mathrm{b}}$ & Range $^{\mathrm{e}}$ \\
& & & & \\
\hline Child-Led Play $^{\mathrm{c}}$ & $83.0 \%$ & $82.8 \%$ & $82.9 \%$ & $70 \%-95 \%$ \\
Positive Composite & $74.5 \%$ & $87.2 \%$ & $80.9 \%$ & $33 \%-100 \%$ \\
Negative Composite & $81.8 \%$ & $89.8 \%$ & $85.8 \%$ & $63 \%-100 \%$ \\
& & & & \\
& & & & \\
Clean-Up & & & & \\
Positive Composite & $79.0 \%$ & $80.9 \%$ & $80.45 \%$ & $66 \%-91.4 \%$ \\
Negative Composite & $77.7 \%$ & $67.9 \%$ & $73.55 \%$ & $50 \%-100 \%$ \\
Effective Commands & $85.7 \%$ & $65.6 \%$ & $72.4 \%$ & $43.6 \%-100 \%$ \\
Contingent Praise & $100 \%$ & $81.8 \%$ & $83.75 \%$ & $75 \%-100 \%$ \\
& & $94.2 \%$ & $97.1 \%$ & $94 \%-100 \%$
\end{tabular}

Note. Interobserver reliability was calculated for 12 out of the 59 5-min DPICS Child-Led Play situations, and 11 out of the 59 5-min DPICS Clean-Up situations during the baseline assessment (pre). Interobserver reliability was calculated for 19 out of the 39 5-min DPICS Child-Led Play situations, and 17 out of the 39 5-min DPICS Clean-Up situations during the post-assessment (post). ${ }^{a}$ Reliability percentages were calculated by summing agreements across participants and dividing by agreements plus disagreements across participants on each variable.

${ }^{\mathrm{b}}$ The overall reliability percentage was calculated by summing each reliability percentage for each listed category (i.e. Child-Led Play, Positive Composite, Negative Composite) and dividing by the total number of categories (8).

${ }^{\mathrm{c}}$ Reliability for the Child-Led Play composite was calculated by summing each overall reliability percentage for each participant and dividing by the total number of participants. The overall reliability percentage calculation included each variable coded for during the DPICS assessment: (a)labeled praises, (b) reflections, (c) behavior descriptions, (d) questions, (e) commands, (f) negative talk, (g) unlabeled praise, and (h) neutral talk. 
${ }^{\mathrm{d}}$ Reliability for the Clean-Up composite was calculated by summing each overall reliability percentage for each participant and dividing by the total number of participants. The overall reliability percentage calculation included each variable coded for during the DPICS assessment: (a)labeled praises, (b) reflections, (c) behavior descriptions, (d) questions, (e) commands, (f) negative talk, (g) unlabeled praise, and (h) neutral talk

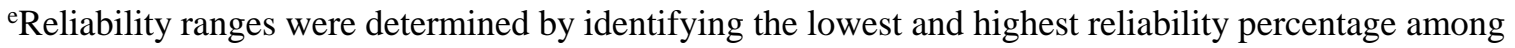
individual participants. 
Table 2

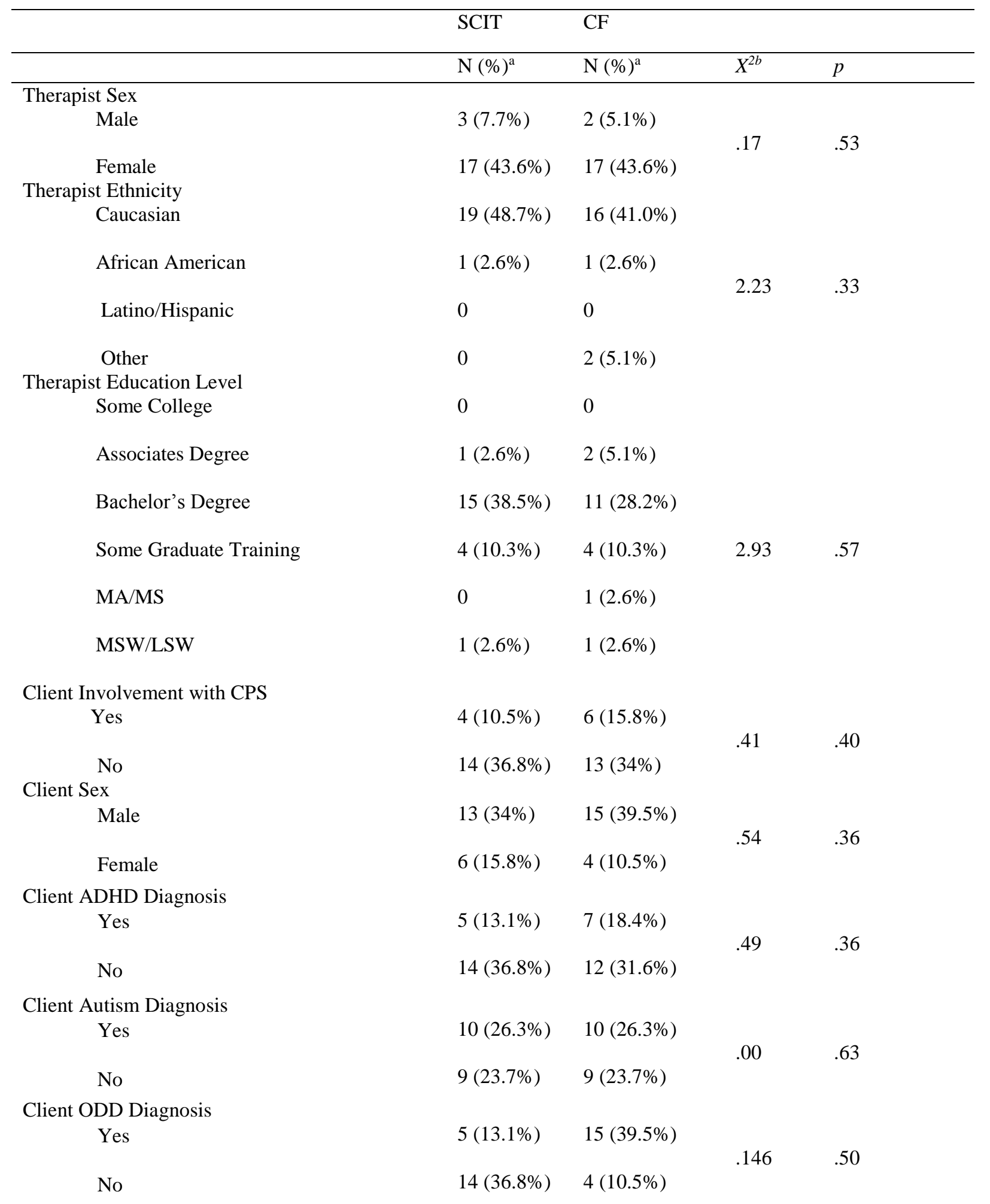


Client CD Diagnosis

Yes

No

Client Disruptive Behavior Disorder Diagnosis Yes

No

Client PTSD Diagnosis

Yes

No

Client ID Diagnosis

Yes

No

Client "Other" Diagnosis

Yes

No
$0(0 \%) \quad 1(2.6 \%)$

$19(50 \%) \quad 18(47.3 \%) \quad 1.02 \quad .50$

$6(15.8 \%) \quad 6(15.8 \%)$

$13(34.2 \%) \quad 13(34.2 \%)$

$1(2.6 \%) \quad 1(2.6 \%)$

$18(47.4 \%) \quad 18(47.4 \%)$

$1(2.6 \%) \quad 1(2.6 \%)$

$18(47.4 \%) \quad 18(47.4 \%)$

.00

$5(13.2 \%) \quad 4(10.5 \%)$

$1436.8 \%) \quad 15(39.5 \%)$

Categorical Variables at Baseline Broken Down by SCIT and Attention Control Groups

Note: $\mathrm{SCIT}=$ Staff Child Interaction Therapy; $\mathrm{CF}=$ Compassion Fatigue

aTotal percentage was calculated for each question by summing the number of responses specific to that question and dividing by the total number of participants.

${ }^{\mathrm{b}} \mathrm{Chi}$ square analyses were run between the SCIT group and CF group to assess differences on categorical variables at the baseline assessment. 
Table 3

Continuous Variables at Baseline Broken Down by SCIT and Attention Control Groups

\begin{tabular}{|c|c|c|c|c|}
\hline & SCIT & $\mathrm{CF}$ & & \\
\hline & $\mathrm{M}(\mathrm{SD})$ & $M(S D)$ & $t^{a}$ & $p$ \\
\hline $\begin{array}{l}\text { Amount of Time } \\
\text { Working with } \\
\text { Client (months) }\end{array}$ & $6.48(7.82)$ & $16.65(24.19)$ & -1.935 & .06 \\
\hline $\begin{array}{l}\text { Hours per Week } \\
\text { with Client }\end{array}$ & $8.07(6.52)$ & $10.06(6.41)$ & 1.09 & .283 \\
\hline Client Age & $5.35(2.07)$ & $5.06(1.48)$ & .552 & .578 \\
\hline $\begin{array}{l}\text { Length of Client } \\
\text { Involvement with } \\
\text { Mental Health } \\
\text { Services }\end{array}$ & $25.33(26.48)$ & $22.44(15.88)$ & .382 & .693 \\
\hline
\end{tabular}

Note. SCIT $=$ Staff-Child Interaction Therapy; $\mathrm{CF}=$ Compassion Fatigue

${ }^{\text {aT }}$-tests were run between the SCIT group and CF group to assess differences on continuous variables at the baseline assessment. 
Table 4

Dependent Variables at Baseline for Child-Led Play DPICS situation

\begin{tabular}{lllll}
\hline & SCIT & CF & & \\
\hline & M (SD) & M (SD) & $t^{c}$ & $p$ \\
\hline $\begin{array}{l}\text { Positive } \\
\text { Composite }\end{array}$ & $3.95(3.14)$ & $2.66(2.16)$ & 2.35 & .07 \\
$\begin{array}{l}\text { Negative } \\
\text { Composite }^{\mathrm{b}}\end{array}$ & $24.35(13.48)$ & $30.58(16.26)$ & -1.31 & .20 \\
\hline
\end{tabular}

Note. SCIT $=$ Staff Child Interaction Therapy; $\mathrm{CF}=\mathrm{Compassion}$ Fatigue

aPositive Composite was calculated by conducting a frequency count of the total number of labeled praises, behavior descriptions, and reflections.

${ }^{b}$ Negative Composite was calculated by a conducting a frequency count of the total number of criticisms, questions, direct and indirect commands.

${ }^{\mathrm{c} T}$-tests were run between the SCIT group and CF group to assess differences on dependent variables at baseline. 


\section{Table 5}

Dependent Variables at Baseline for Clean-Up DPICS Situation

\begin{tabular}{lllll}
\hline & SCIT & CF & & \\
\hline & M (SD) & M(SD) & $t^{e}$ & $p$ \\
\hline Positive Composite $^{\mathrm{a}}$ & $2.16(2.01)$ & $1.95(1.61)$ & -.45 & .66 \\
$\begin{array}{l}\text { Negative } \\
\text { Composite }\end{array}$ & $13.21(8.48)$ & $13.90(7.28)$ & -.55 & .58 \\
$\begin{array}{l}\text { Effective } \\
\text { Commands }\end{array}$ & $6.52(9.68)$ & $4.10(11.57)$ & .66 & .55 \\
Contingent Praise $^{\mathrm{d}}$ & 0 & 0 & - & - \\
\hline
\end{tabular}

Note. $\mathrm{SCIT}=$ Staff Child Interaction Therapy; $\mathrm{CF}=$ Compassion Fatigue

aPositive composite was calculated by a conducting a frequency count of the total number of labeled praises, behavior descriptions, and reflections.

${ }^{b}$ Negative Composite was calculated by a conducting a frequency count of the total number of criticisms, questions, and indirect commands.

'Effective Commands were calculated by summing the total number of direct commands and dividing by the total number of commands.

${ }^{\mathrm{d} C}$ Contingent Praise was calculated by determining a frequency count of the number of labeled praises that directly followed compliance.

${ }^{\mathrm{e}} \mathrm{T}$-tests were run between the SCIT group and CF group to assess differences on dependent variables at baseline 
Table 6

Dependent Variables Broken Down by SCIT and Compassion Fatigue Groups for the Child-Led Play DPICS Situation

\begin{tabular}{|c|c|c|c|c|c|c|c|c|c|c|c|c|c|c|c|c|c|}
\hline & & & Pre & & & & Post & & & & $\begin{array}{l}\text { Time } \\
\text { X } \\
\text { Group }\end{array}$ & & Time $^{\mathrm{d}}$ & & Groul & & \\
\hline \multirow{4}{*}{$\begin{array}{l}\text { Positive } \\
\text { Composite }^{a}\end{array}$} & Group & $n$ & M & $S D$ & $S E$ & $n$ & $\mathrm{M}$ & $S D$ & $S E$ & $F(1,37)$ & $p$ & $d^{c}$ & $F(1,37) \quad p$ & $d$ & $F(1,37)$ & $p$ & $d$ \\
\hline & & & & & & & & & & $49.68 * *$ & .00 & .573 & $46.280 * * .00$ & .556 & $54.427 * *$ & .00 & .595 \\
\hline & SCIT & 20 & 3.95 & 3.14 & .70 & 20 & 21.21 & 10.97 & 2.45 & & & & & & & & \\
\hline & $\mathrm{CF}$ & 19 & 2.65 & 2.16 & .50 & 19 & 3.65 & 7.55 & 1.732 & & & & & & & & \\
\hline \multirow[t]{3}{*}{$\begin{array}{l}\text { Negative } \\
\text { Composite }^{\mathrm{b}}\end{array}$} & & & & & & & & & & $10.93 *$ & .002 & .228 & $38.153 * * .00$ & .508 & $15.437 * *$ & .00 & .294 \\
\hline & SCIT & 20 & 24.35 & 13.48 & 3.01 & 20 & 5.40 & 7.03 & 1.57 & & & & & & & & \\
\hline & $\mathrm{CF}$ & 19 & 30.58 & 16.26 & 3.73 & 19 & 22.63 & 10.69 & 2.45 & & & & & & & & \\
\hline
\end{tabular}

Note. Raw scores are reported. SCIT= Staff-Child Interaction Therapy; $\mathrm{CF}=\mathrm{Compassion}$ Fatigue

aPositive composite was calculated by a conducting a frequency count of the total number of labeled praises, behavior descriptions, and reflections.

begative Composite was calculated by a conducting a frequency count of the total number of criticisms, questions, direct and indirect commands.

'Cohen's d=effect size between SCIT and CF groups at the post-assessment phase based on changes in mean scores from pre-to post-assessment. Cohen's $\mathrm{D}$ is the typical measure of effect size across PCIT literature.

dThe F-value, effect size (Cohen's D), and p-value for the main effect of the Time condition are presented.

'The F-value, effect size (Cohen's D), and p-value for the main effect of the Group condition are presented.

$* p<.05 . * * p<.01$. 
Table 7

Dependent Variables Broken Down by SCIT and Compassion Fatigue Groups for the Clean-Up DPICS Situation

\begin{tabular}{|c|c|c|c|c|c|c|c|c|c|c|c|c|c|c|c|c|c|c|}
\hline & & & Pre & & & & Post & & & & $\begin{array}{l}\text { Time X } \\
\text { Group }\end{array}$ & & Tin & $n e^{f}$ & & Groul & & \\
\hline & Group & $\mathrm{n}$ & $M$ & $S D$ & $S E$ & $\mathrm{n}$ & $\mathrm{M}$ & $S D$ & $S E$ & $F(1,37)$ & $p$ & $d^{e}$ & $F(1,37)$ & $p$ & $d$ & $F(1,37)$ & $p$ & $d$ \\
\hline \multirow{3}{*}{$\begin{array}{l}\text { Positive } \\
\text { Composite }^{\mathrm{a}}\end{array}$} & & & & & & & & & & 1.932 & .173 & .45 & .390 & .536 & .201 & .384 & .539 & .201 \\
\hline & SCIT & 20 & 2.16 & 2.01 & .45 & 20 & 2.68 & 2.65 & .59 & & & & & & & & & \\
\hline & $\mathrm{CF}$ & 19 & 1.95 & 1.61 & .37 & 19 & 1.45 & 1.40 & .32 & & & & & & & & & \\
\hline \multirow{3}{*}{$\begin{array}{l}\text { Negative } \\
\text { Composite }^{\mathrm{b}}\end{array}$} & & & & & & & & & & $5.998^{*}$ & 0.019 & .80 & 44.02 & .000 & .79 & 5.825 & .021 & .79 \\
\hline & SCIT & 20 & 13.21 & 8.48 & 1.90 & 20 & 3.32 & 4.16 & 2.9 & & & & & & & & & \\
\hline & $\mathrm{CF}$ & 19 & 13.90 & 7.28 & 1.70 & 19 & 10.55 & 5.90 & 1.35 & & & & & & & & & \\
\hline \multirow{3}{*}{$\begin{array}{l}\text { Effective } \\
\text { Commands }^{\mathrm{c}}\end{array}$} & & & & & & & & & & .978 & .329 & .33 & 2.71 & .108 & .54 & 2.23 & .143 & .49 \\
\hline & SCIT & 20 & 6.52 & 9.68 & 2.16 & 20 & 15.19 & 24.42 & 5.46 & & & & & & & & & \\
\hline & $\mathrm{CF}$ & 19 & 4.10 & 11.57 & 2.70 & 19 & 1.64 & 6.04 & 1.39 & & & & & & & & & \\
\hline \multirow{3}{*}{$\begin{array}{l}\text { Contingent } \\
\text { Praise }^{\mathrm{d}}\end{array}$} & & & & & & & & & & 3.04 & .090 & .57 & 3.04 & .090 & .57 & 3.04 & .090 & .57 \\
\hline & SCIT & 20 & 0 & 0 & 0 & 20 & 1.16 & 2.67 & .61 & & & & & & & & & \\
\hline & $\mathrm{CF}$ & 19 & 0 & 0 & 0 & 19 & 0 & 0 & 0 & & & & & & & & & \\
\hline
\end{tabular}

Note. Raw sores are reported. SCIT= Staff-Child Interaction Therapy; $\mathrm{CF}=\mathrm{Compassion}$ Fatigue

aPositive composite was calculated by a conducting a frequency count of the total number of labeled praises, behavior descriptions, and reflections.

${ }^{b}$ Negative Composite was calculated by a conducting a frequency count of the total number of criticisms, questions, direct and indirect commands. 
${ }^{c}$ Effective Commands were calculated by summing the total number of direct commands and dividing by the total number of commands.

${ }^{\mathrm{d} C o n t i n g e n t ~ P r a i s e ~ w a s ~ c a l c u l a t e d ~ b y ~ d e t e r m i n i n g ~ a ~ f r e q u e n c y ~ c o u n t ~ o f ~ t h e ~ n u m b e r ~ o f ~ l a b e l e d ~ p r a i s e s ~ t h a t ~ d i r e c t l y ~ f o l l o w e d ~ c o m p l i a n c e . ~}$

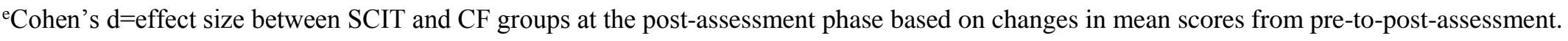

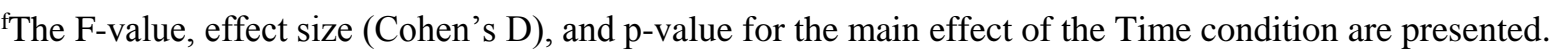

'The F-value, effect size (Cohen's D), and p-value for the main effect of the Group condition are presented.

$* p<.05$ 
Figure 1. Positive Composite Variable by Group from Pre- to 6-to-8 weeks Post Assessment during Child-Led Play Situation, Including Post-Hoc Tukey HSD Tests

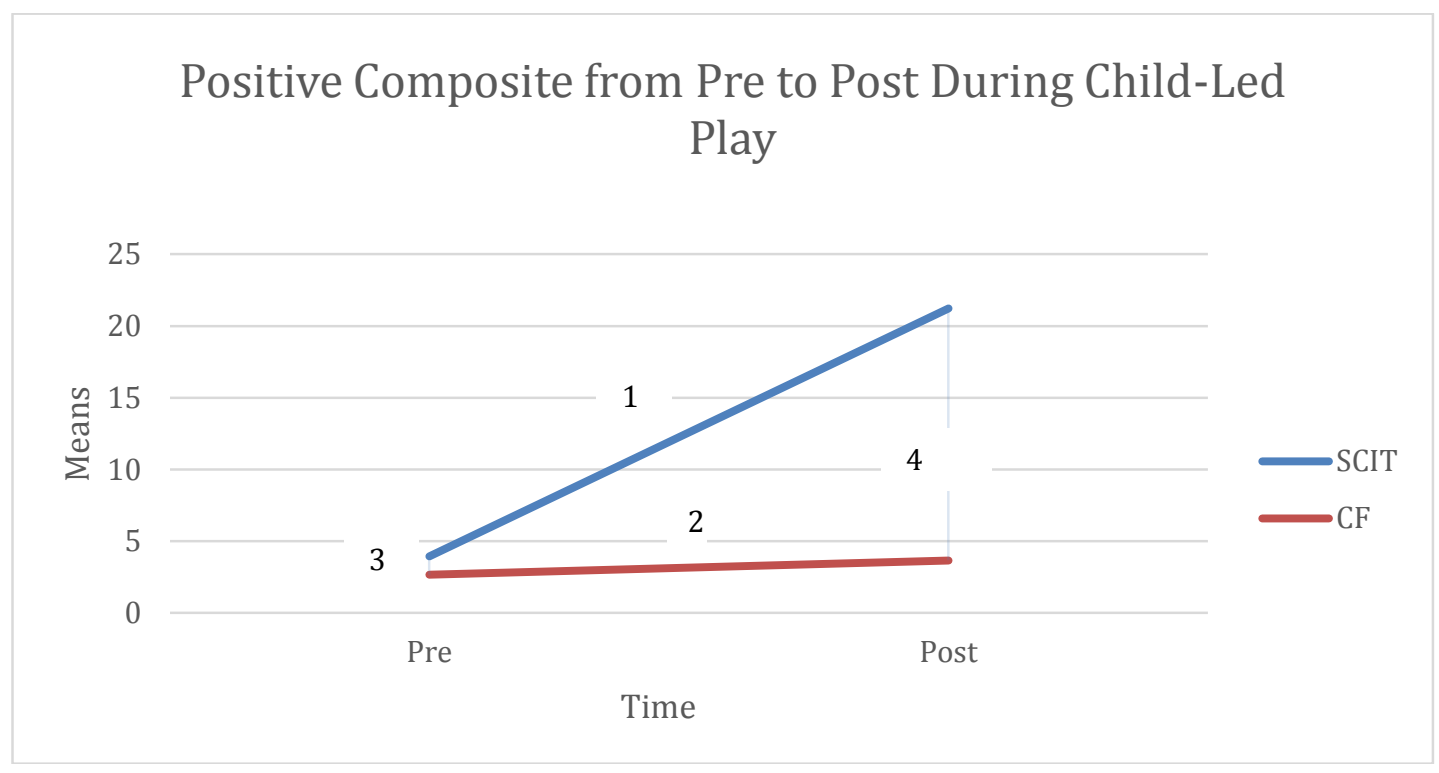

1. Post hoc comparisons using the Tukey HSD test indicated that the mean score for the SCIT group at pre-treatment $(\mathrm{M}=3.95, \mathrm{SD}=3.14)$ was significantly different than the mean score for the SCIT group at post-treatment $(\mathrm{M}=21.21, \mathrm{SD}=10.97), \mathrm{p}<.01$. 2. Post hoc comparisons using the Tukey HSD test indicated that the mean score for the attention control $(\mathrm{CF})$ group at pre-treatment $(\mathrm{M}=2.65, \mathrm{SD}=2.16)$ was not significantly different than the mean score for the attention control group at post-treatment $(\mathrm{M}=3.65$ $\mathrm{SD}=7.55), \mathrm{p}>.05$.

3. Post hoc comparisons using the Tukey HSD test indicated that the mean score for the SCIT group at pre-treatment $(\mathrm{M}=3.95, \mathrm{SD}=3.14)$ was not significantly different than the mean score for the attention control $(\mathrm{CF})$ group at pre-treatment $(\mathrm{M}=2.65, \mathrm{SD}=$ 2.16), $\mathrm{p}>.05$.

4. Post hoc comparisons using the Tukey HSD test indicated that the mean score for the SCIT group at post-treatment $(\mathrm{M}=21.21, \mathrm{SD}=10.97)$ was significantly higher than the mean score for the attention control $(\mathrm{CF})$ group at post-treatment $(\mathrm{M}=3.65, \mathrm{SD}=7.55)$, $\mathrm{p}<.01$. 
Figure 2. Negative Composite Variable by Group from Pre- to 6-to-8 weeks Post Assessment during Child-Led Play Situation, Including Post-Hoc Tukey HSD Tests

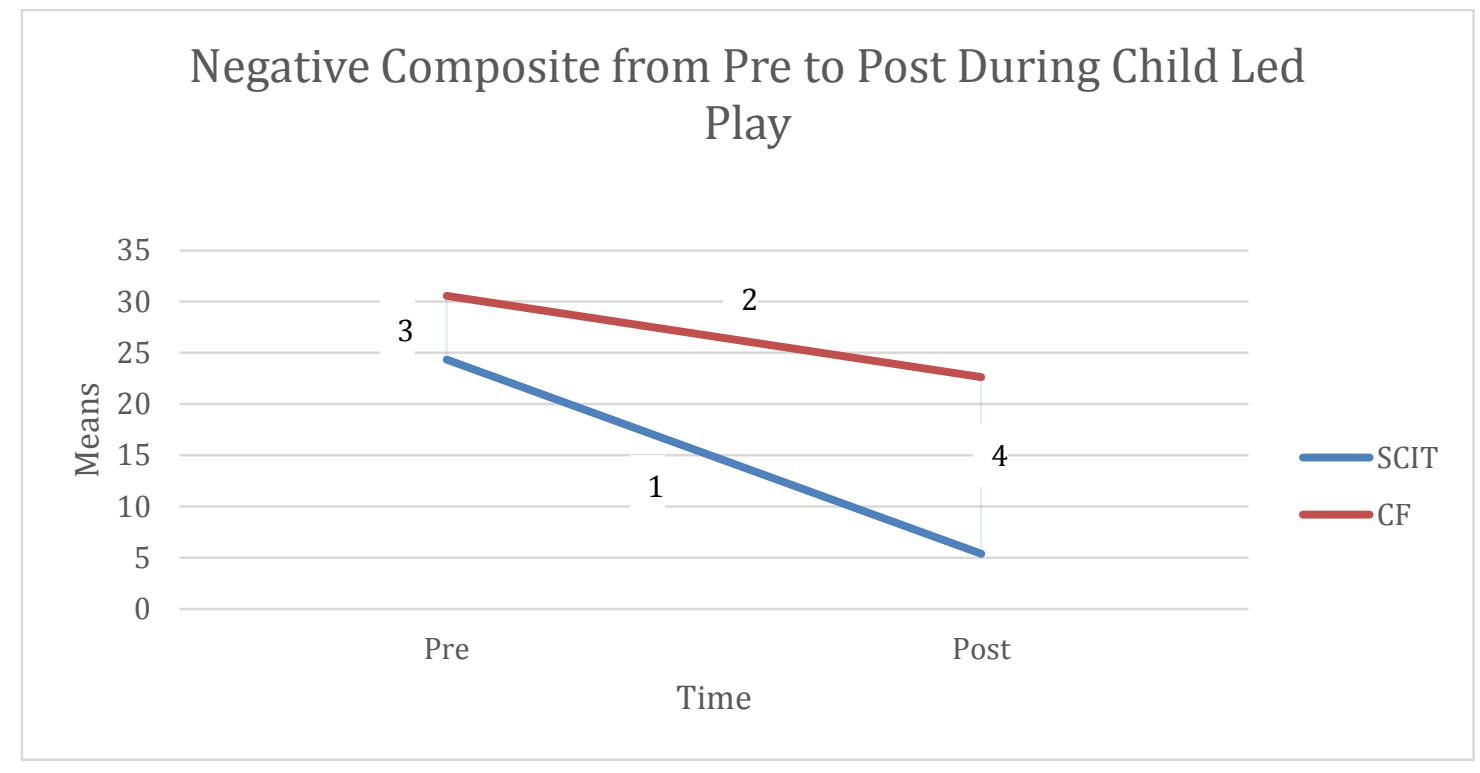

Figure 2. T-tests indicated that differences in the negative composite variable between the SCIT and attention control group at baseline were not significant.

1. Post hoc comparisons using the Tukey HSD test indicated that the mean score for the SCIT group at pre-treatment $(M=24.35, \mathrm{SD}=13.48)$ was significantly different than the mean score for the SCIT group at post-treatment $(\mathrm{M}=5.40, \mathrm{SD}=7.03), \mathrm{p}<.01$.

2. Post hoc comparisons using the Tukey HSD test indicated that the mean score for the attention control $(\mathrm{CF})$ group at pre-treatment $(\mathrm{M}=30.58, \mathrm{SD}=16.26)$ was significantly different than the mean score for the attention control group at post-treatment $(M=22.63$, $\mathrm{SD}=10.69), \mathrm{p}>.05$.

3. Post hoc comparisons using the Tukey HSD test indicated that the mean score for the SCIT group at pre-treatment $(\mathrm{M}=24.35, \mathrm{SD}=13.48)$ was not significantly different than the mean score for the attention control $(\mathrm{CF})$ group at pre-treatment $(\mathrm{M}=30.58, \mathrm{SD}=$ 16.26), $\mathrm{p}>.05$.

4. Post hoc comparisons using the Tukey HSD test indicated that the mean score for the SCIT group at post-treatment $(\mathrm{M}=5.40, \mathrm{SD}=7.03)$ was significantly higher than the mean score for the attention control $(\mathrm{CF})$ group at post-treatment $(\mathrm{M}=22.63, \mathrm{SD}=$ $10.69), \mathrm{p}<.01$. 
Figure 3. Negative Composite Variable by Group from Pre- to 6-to-8 weeks Post Assessment during Clean Up Situation, Including Post-Hoc Tukey HSD Tests

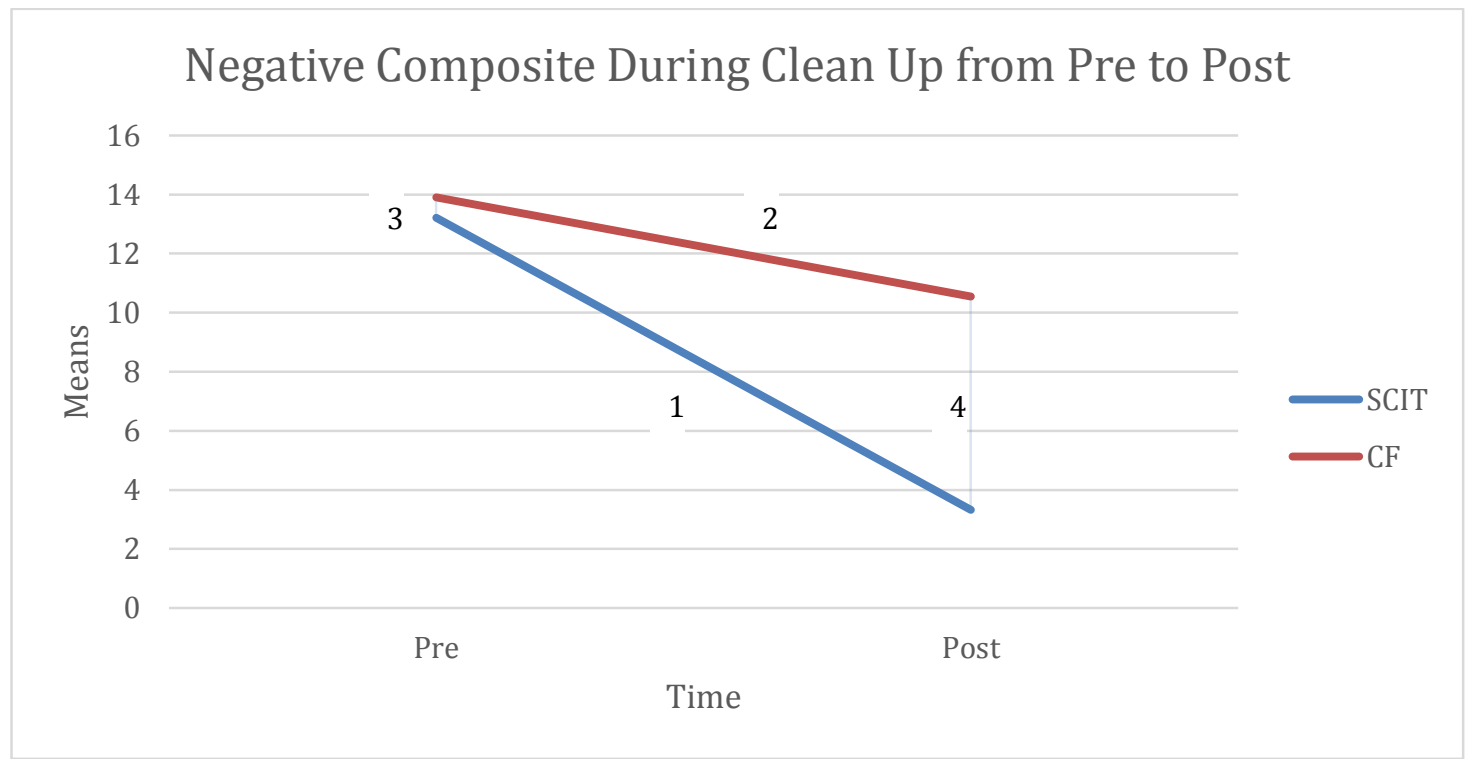

Figure 3. T-tests indicated that differences in the negative composite variable between the SCIT and attention control group at baseline were not significant.

1. Post hoc comparisons using the Tukey HSD test indicated that the mean score for the SCIT group at pre-treatment $(\mathrm{M}=13.21, \mathrm{SD}=8.48)$ was significantly different than the mean score for the SCIT group at post-treatment $(\mathrm{M}=3.32 \mathrm{SD}=4.16), \mathrm{p}<.01$.

2. Post hoc comparisons using the Tukey HSD test indicated that the mean score for the attention control $(\mathrm{CF})$ group at pre-treatment $(\mathrm{M}=13.90, \mathrm{SD}=7.28)$ was not significantly different than the mean score for the attention control group at posttreatment $(\mathrm{M}=10.55, \mathrm{SD}=5.90), \mathrm{p}>.05$.

3. Post hoc comparisons using the Tukey HSD test indicated that the mean score for the SCIT group at pre-treatment $(\mathrm{M}=13.21, \mathrm{SD}=8.48)$ was not significantly different than the mean score for the attention control $(\mathrm{CF})$ group at pre-treatment $(\mathrm{M}=13.90, \mathrm{SD}=$ 7.28), $\mathrm{p}>.05$.

4. Post hoc comparisons using the Tukey HSD test indicated that the mean score for the SCIT group at post-treatment $(\mathrm{M}=3.32, \mathrm{SD}=4.16)$ was significantly higher than the mean score for the attention control $(\mathrm{CF})$ group at post-treatment $(\mathrm{M}=10.55, \mathrm{SD}=5.90)$, $\mathrm{p}<.01$. 
Appendix A

SCIT vs. PCIT

\begin{tabular}{|c|c|c|}
\hline & $\begin{array}{l}\text { Parent-Child Interaction Therapy } \\
\text { (PCIT) }\end{array}$ & $\begin{array}{l}\text { Staff-Child Interaction Therapy } \\
\text { (SCIT) }\end{array}$ \\
\hline Training Requirements & $\begin{array}{l}\text { - } 56 \text { hours of training } \\
\text { - } 5 \text { day PCIT training } \\
\text { - } 2 \text { day advanced training } \\
\text { - } 1 \text { year } \\
\text { - } 2 x / \text { month consultation } \\
\text { - } \text { Completion of full cases } \\
\text { - Master's degree }\end{array}$ & $\begin{array}{l}\text { - } 2 \text { day workshop } \\
\text { - } 3 \text { consultations calls } \\
\text { before } 2^{\text {nd }} \text { workshop } \\
\text { - Bachelors or Masters degree }\end{array}$ \\
\hline Setting & Outpatient setting & Home-based service \\
\hline Clients & 2-9 years of age & 2-9 years of age \\
\hline Standard session & $\begin{array}{l}\text { Typically takes place in outpatient } \\
\text { setting } \\
\text { Coaching via bug-in-ear device and } \\
\text { microphone system behind one-way } \\
\text { mirror } \\
\text { Modeling of skills }\end{array}$ & $\begin{array}{l}\text { Takes place in home of client } \\
\text { No coaching } \\
\text { Modeling of skills } \\
\text { Coding of skills with feedback }\end{array}$ \\
\hline Coding & CDI and PDI coding & CDI and PDI coding \\
\hline Child-Led Phase & $\begin{array}{l}\text { Child-Directed Interaction (CDI) } \\
\text { Parent learns/implements PRIDE } \\
\text { skills through therapist coaching }\end{array}$ & $\begin{array}{l}\text { Child-Directed Interaction (CDI) } \\
\text { Therapist learns/implements PRIDE } \\
\text { skills and models for parent }\end{array}$ \\
\hline $\begin{array}{l}\text { Parent/Adult Led } \\
\text { Phase }\end{array}$ & $\begin{array}{l}\text { Parent-Directed Interaction (PDI) } \\
\text { Timeout sequence involves timeout } \\
\text { chair and back-up room }\end{array}$ & $\begin{array}{l}\text { Adult-Directed Interaction (ADI) } \\
\text { Broken record technique } \\
\text { Physical guide } \\
\text { Restriction of privilege }\end{array}$ \\
\hline
\end{tabular}




\section{Appendix B}

\section{ADI Diagram with ADI Script}

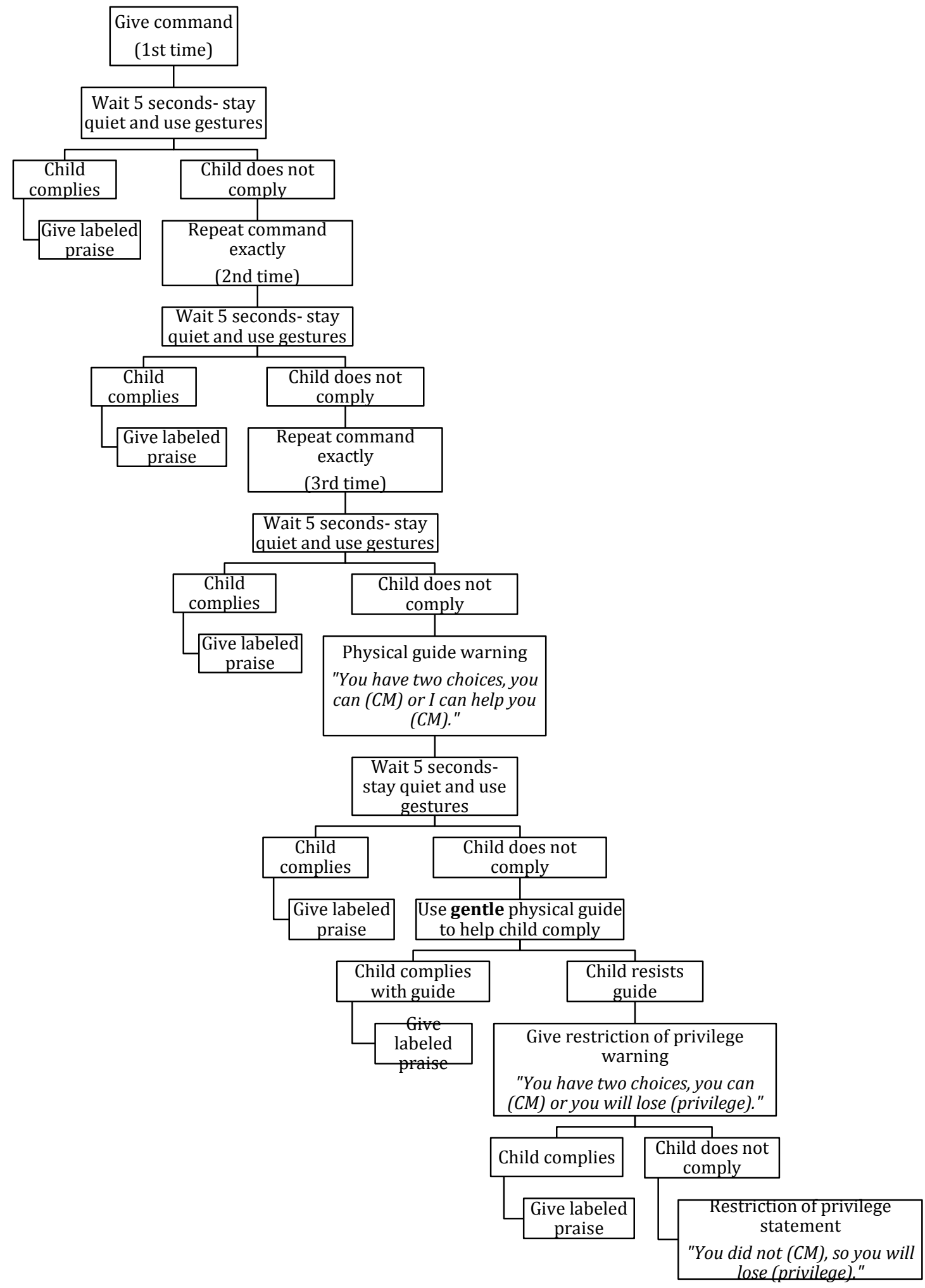


Appendix C

SCIT Workshop Target Skills

\begin{tabular}{|c|c|c|}
\hline Behavior Category & Definition & Example \\
\hline Labeled Praise & $\begin{array}{l}\text { A specific statement of } \\
\text { approval that teaches the } \\
\text { child what can be done to } \\
\text { receive further approval }\end{array}$ & $\begin{array}{l}\text { "You did a great job of } \\
\text { building the tower." }\end{array}$ \\
\hline Reflection & $\begin{array}{l}\text { A declarative phrase or } \\
\text { statement that has the same } \\
\text { meaning as a child } \\
\text { verbalization }\end{array}$ & $\begin{array}{l}\text { Child: "That's a funny } \\
\text { clown." } \\
\text { TSS: "You think he's } \\
\text { funny." }\end{array}$ \\
\hline Behavior Description & $\begin{array}{l}\text { A non-evaluative, } \\
\text { declarative sentence or } \\
\text { phrase in which the subject } \\
\text { is the child and the verb } \\
\text { describes the child's } \\
\text { ongoing or immediately } \\
\text { completed behavior. }\end{array}$ & $\begin{array}{l}\text { Child: (colors in flower) } \\
\text { TSS: "You're coloring the } \\
\text { flower red." }\end{array}$ \\
\hline Contingent Praise & $\begin{array}{l}\text { A specific statement of } \\
\text { approval directly following } \\
\text { compliance }\end{array}$ & $\begin{array}{l}\text { TSS: "Please hand me the } \\
\text { red block." } \\
\text { (Child hands TSS the } \\
\text { block) } \\
\text { TSS: "Thank you so much } \\
\text { for handing me the red } \\
\text { block." }\end{array}$ \\
\hline Effective Commands & $\begin{array}{l}\text { A direct, singular statement } \\
\text { that contains an order or } \\
\text { direction for a behavior } \\
\text { from the child }\end{array}$ & $\begin{array}{l}\text { TSS: "Please pick up the } \\
\text { yellow crayon." }\end{array}$ \\
\hline
\end{tabular}

Note: Definitions for these categories were based on the DPICS III: Eyberg, S. M., Nelson, M. M., Duke, M., \& Boggs, S. R. (2005). Manual for the Dyadic Parent-Child Interaction Coding System (3rd ed.), Unpublished manuscript, University of Florida, Gainesville. 
Appendix D

DPCIS Coding Sheet

\begin{tabular}{|c|c|c|c|}
\hline Skill & Tally & Number & Mastery \\
\hline Labeled Praise & & & 10 \\
\hline Reflections & & & 10 \\
\hline Descriptions & & & 10 \\
\hline Imitation & Satisfactory $\quad$ Needs Practice & & \\
\hline Enjoyment & Needs Practice & & \\
\hline $\begin{array}{l}\text { Unlabeled } \\
\text { Praise }\end{array}$ & & & \\
\hline Neutral Talk & & & \\
\hline Negative Talk & & & \multirow{3}{*}{$\begin{array}{l}3 \text { or fewer } \\
\text { combined }\end{array}$} \\
\hline Commands & & & \\
\hline Questions & & & \\
\hline
\end{tabular}


Appendix E

Child Behavior Checklist

- Compliance with 30-70\% of commands

- 15 Neutral Statements

- Majority of Neutral Statements

- 1 Annoying or obnoxious sequence lasting 15-45 secs -[CHOOSE 1]

○ Tantrum

○ Toy tapping

○ Repeating "Bad word"

- Burping/Farting Noises

- Throwing Toy on floor

- Scribble on paper

o Yell, scream, squeak

- Play with truck

- Share toy

- Color with Crayon

- Build Tower

- Build Fence

- Pretend to eat food

- Laugh

- Sing a 1-line song

- Make animal noise

- Say Statement about Self: "I'm funny!" or "I'm silly worm!"

- Say Statement that is Incorrect: "I've got 3 eyes!" or "My hair is blue!"

- Say Statement about Toy: "Car go vroom vroom vroom" or "Horsies go Neeeigh!"

- Say Mistake statement: “Oopsies! Or Ought O Spaghettis!”

- Ask a question

- Show parent toy

- Tell parent what you are doing

- Talk about fence

- Talk about Tower

- Talk about favorite color

Tempel A. T., McNeil, C. B., Chengappa, K., \& Costello, A. H. (2014). Evaluation of an existing parenting class within a women's state correctional facility and a parenting class modeled from Parent-Child Interaction Therapy. Children and Youth Services Review, 46, 238-44 


\section{Appendix F}

\section{Treatment as Usual Discussion Questions}

\section{Treatment as Usual (TAU) Fidelity Discussion}

\section{Day to day activities (conducting a session)}

On average, how long (Days? Hours?) do you spend with a consumer before they terminate?

What are the top (3? 5?) treatment goals for your consumers?

How do you measure consumer progress?

What sort of data do you collect for a consumer? What does this data look like? How often do you collect this data?

What does a typical session look like with this consumer?

Walk us through: first 15 minutes, second 15 minutes?

Can you describe, in detail, your last session with your consumer?

What other variables could change how you conduct a session?

Do you ever leave the home with the consumer and his/her family? How often?

Where do you go? What do you do?

How long do you usually see a consumer (total months/years).

How would you describe your role in the life of your consumer?

What determines when services conclude? How have cases concluded that you were a TSS for? How many cases have you had that you would say have "gotten better" and no longer needed help?

What percentage of the session is spent working with the parent and child together?

What percent is working with just the parent? Just the child?

\section{Challenges in the home}

What are some common distractions in the home that keep you from completing your goals for a session? How often do these distractions arise?

Describe the involvement the caregivers of your consumers have in a typical session (think back to your last session). How much time the caregiver was there (i.e., in the room- actively involved with what you were doing with the client)/How much time were you there?

What sorts of challenges arise when working with consumers?

When challenges arise, what do you typically do about it?

If a child were to become very aggressive toward you or the parent, what do you do?

If a child does not listen to you or the parent (in front of you) what do you do about it?

\section{Supervision}

How often and for how long do you receive supervision?

What percentage of the time that you're in the home of your consumer is your supervisor also in the home?

What do you talk about in supervision? For how long? 


\section{Appendix G}

\section{Control Workshop Protocol}

\begin{tabular}{|c|c|}
\hline 30 minutes & $\begin{array}{l}\text { Explanation of informed consent and measures by graduate researcher } \\
\text { Intro to training, trainers, trainees }\end{array}$ \\
\hline 50 minutes & $\begin{array}{l}\text { Baseline assessment with confederate child using the DPICS } \\
\text { Completion of measures }\end{array}$ \\
\hline 90 minutes & Breakout discussion groups \\
\hline 60 minutes & Lunch \\
\hline 60 minutes & $\begin{array}{l}\text { Compassion fatigue presentation } \\
\text { - Compassion fatigue prevention discussion } \\
\text { - Explanation of job burnout } \\
\text { - Video demonstration } \\
\end{array}$ \\
\hline 45 minutes & Discussion-based game \\
\hline 30 minutes & $\begin{array}{l}\text { Reflection } \\
\text { Satisfaction survey }\end{array}$ \\
\hline
\end{tabular}


Appendix $\mathrm{H}$

SCIT Workshop Protocol

\begin{tabular}{|c|c|}
\hline 30 minutes & $\begin{array}{l}\text { Explanation of informed consent and measures by graduate researcher } \\
\text { Intro to training, trainers, trainees }\end{array}$ \\
\hline 50 minutes & $\begin{array}{l}\text { Baseline assessment with confederate child using the DPICS/ } \\
\text { Completion of measures }\end{array}$ \\
\hline 45 minutes & $\begin{array}{l}\text { SCIT vs. PCIT explanation } \\
\text { CDI Teach } \\
\text { Explanation/Rationale for CDI } \\
\text { - PRIDE skills taught } \\
\text { - PRIDE skills modeled } \\
\text { - Planned ignoring taught } \\
\text { - Planned ignoring modeled }\end{array}$ \\
\hline 30 minutes & $\begin{array}{l}\text { DPICS training } \\
\text { - Live demonstrations in between slides }\end{array}$ \\
\hline 60 minutes & $\begin{array}{l}\text { CDI Breakout groups } \\
\text { - } \text { CDI practice } \\
\text { - Coding practice } \mathrm{w} / \text { reliability checks }\end{array}$ \\
\hline 60 minutes & $\begin{array}{l}\text { Lunch } \\
\text { • DPICS quizzes completed }\end{array}$ \\
\hline 60 minutes & $\begin{array}{l}\text { ADI teach } \\
\text { Explanation/Rationale for ADI } \\
\text { - Effective commands taught } \\
\text { - Broken record technique taught } \\
\text { - Broken record technique modeled } \\
\text { ADI Coding training }\end{array}$ \\
\hline 60 minutes & $\begin{array}{l}\text { ADI Breakout groups } \\
\text { - } \text { ADI practice } \\
\text { - } \text { ADI coding practice }\end{array}$ \\
\hline 30 minutes & Explanation of SCIT In-home session procedures \\
\hline 30 minutes & $\begin{array}{l}\text { Reflection on training } \\
\text { TAI Assessment }\end{array}$ \\
\hline
\end{tabular}


Appendix I

Mastery Criteria

\begin{tabular}{|l|l|}
\hline Variable & Mastery Criteria \\
\hline Child-Directed Interaction (CDI) & $\begin{array}{l}\text { Provide 10 labeled praised, 10 Behavior } \\
\text { Descriptions, 10 Reflections, and use less } \\
\text { than 3 combined questions, commands, } \\
\text { and/or negative talk/criticism during 5- } \\
\text { minute CDI role-plays with confederate } \\
\text { child }\end{array}$ \\
\hline CDI Coding & $\begin{array}{l}\text { Achieve 80\% coding reliability with } \\
\text { primary researcher during 5-minute CDI } \\
\text { role-plays }\end{array}$ \\
\hline Adult- Directed Interaction (ADI) & $\begin{array}{l}\text { Provide 75\% effective commands and } \\
\text { 75\% effective follow through with the use } \\
\text { of broken-record technique during 5- } \\
\text { minute ADI role-plays with confederate } \\
\text { child }\end{array}$ \\
\hline DPICS Mastery & $\begin{array}{l}\text { Obtain a score of 80\% or higher on one } \\
\text { DPICS quiz }\end{array}$ \\
\hline
\end{tabular}


Appendix $\mathbf{J}$

TSS Demographic Form

\section{Therapeutic Support Staff Demographic Form}

What is your sex?

Male Female

What is your race/ ethnicity?

Caucasian

African American

Latino/ Hispanic

American Indian

Other:

(Please list.)

Years in current position:

Highest degree earned: Some college

Associates degree

Bachelor's degree

Some graduate training

M. A. / M. S.

M. S. W. / L. S. W

Other:

(Please list)

Are you a BCBA (Board certified behavior analyst)?

Yes

No

Are you a BCaBA (Board Certified assistant Behavior Analyst)? 
Yes No

Are you a RBT (Registered Behavior Technician)?

Yes No

Have you ever received training that included coding of skill use and feedback based on coding of skill use in the past?

Yes No

Have you ever heard of Parent-Child Interaction Therapy?

Yes No

Have you received training in Parent-Child Interaction Therapy in the past?

Yes $\quad$ No

Have you ever observed a Parent-Child Interaction Therapy session?

Yes No

Please describe all training you have received at Family Behavioral Resources or elsewhere in the form of a workshop and list the length of the training (please consider workshops on therapeutic techniques and behavior modification).

Workshop Title

Length

Please describe all training you have received at Family Behavioral Resources or elsewhere in the form of a class and list the length of the training (please consider classes on therapeutic techniques and behavior modification)

Workshop Title Length 
How long have you worked with your client?

How many hours do you typically spend with your client each week?

Has your client been involved with Child Protective Services/ Child and Youth Services in the past?

Yes No

Is your client currently involved with Child Protective Services/ Child and Youth Services?

Yes No

What mental disorder(s) has your client been diagnosed with? Please check all that apply.

$\square$ Autism Spectrum Disorder

$\square$ Attention Deficit Hyperactivity Disorder

$\square$ Oppositional Defiant Disorder

$\square$ Conduct Disorder

$\square$ Disruptive Behavior Disorder Not Otherwise Specified

$\square$ Post-Traumatic Stress Disorder

$\square$ Intellectual Disability

$\square$ Other Diagnosis (Please list below.) 
Appendix K

Variables Coded during Standard DPICS Situations

\begin{tabular}{|l|l|l|}
\hline DPCIS Variables being Coded & Definitions & Applicable Situations \\
\hline Positive Parenting Composite & $\begin{array}{l}\text { Labeled praise, reflections, } \\
\text { behavior descriptions, positive } \\
\text { touches }\end{array}$ & Both DPICS situations \\
\hline Negative Parenting Composite & $\begin{array}{l}\text { Questions, negative talk, } \\
\text { commands }\end{array}$ & Both DPICS situations \\
\hline Percentage of Effective & $\begin{array}{l}\text { Total number of effective } \\
\text { commands (those stated directly, } \\
\text { positively, and are specific and } \\
\text { singular) divided by total number } \\
\text { of commands }\end{array}$ & Clean-Up \\
\hline Contingent Praise for Child & $\begin{array}{l}\text { A specific statement of approval } \\
\text { directly following compliance }\end{array}$ & Clean-Up \\
\hline
\end{tabular}




\section{Appendix L \\ Instructions for the Two Standard DPICS Observations}

\section{Child-Led Play}

"In this situation tell that he/she may play with whatever he/she chooses. Let him/her choose any activity he/she wishes. You just follow his/her lead and play along with him/her."

Clean-Up

"That was fine. Now please tell that special playtime is over and the toys must be put away. Make sure you have him/her put the toys away by him/herself. Have him/her put all the toys back in the bag 